\title{
Stability analysis of random nonlinear systems with time-varying delay and its application
}

\author{
Liqiang Yao, Weihai Zhang*
}

\begin{abstract}
This paper studies a class of random nonlinear systems with time-varying delay, in which the $r$-order moment $(r \geq 1)$ of the random disturbance is finite. Firstly, some general conditions are proposed to guarantee the existence and uniqueness of the global solution to random nonlinear timedelay systems. Secondly, some definitions and criteria on noiseto-state stability in the moment sense and in probability sense are given by Lyapunov method respectively. Finally, two regulation controllers are constructed respectively for two corresponding random nonlinear time-delay systems and the effectiveness of two proposed recursive procedures are demonstrated by two simulation examples.
\end{abstract}

Index Terms-Random nonlinear systems, time-varying delay, regulation controller, noise-to-state stability.

\section{INTRODUCTION}

Time-delay is widespread in many actual systems such as network transmission systems, hydraulic systems and power systems. The time-delay phenomenon is often caused by the inherent characteristics of physical systems such as communication system and mechanical drive system. In addition, the devices of practical systems (for instance, controllers and actuators) need time to complete operations during the running process of systems, which inevitably makes the time-delay phenomenon. The existence of time-delay phenomenon can affect system performance seriously and brings difficulties for system analysis and synthesis. Nevertheless, time-delay phenomenon sometimes can be used to improve the control performance of some systems such as repetitive control systems. Therefore, it is of great significance to study how to eliminate and utilize time-delay phenomena. Over the past few decades, many scholars have committed themselves to studying timedelay systems in theory and engineering applications ([1] 3]).

On the other hand, many practical systems in engineering, which are often subject to random disturbance from external environment, are modeled as stochastic nonlinear systems or random nonlinear systems (44,7). The difference lies in the random disturbance which appeared in the former is a white noise process (i.e., the derivative of Wiener process), while

* Corresponding author.

This work was supported by National Natural Science Foundation of China (Nos: 61573227, 61633014), SDUST Research Fund (No. 2015TDJH105), Research Fund for the Taishan Scholar Project of Shandong Province of China

Liqiang Yao is with the College of Electrical Engineering and Automation, Shandong University of Science and Technology, Qingdao, Shandong Province, 266590, P. R. China and the School of Mathematics and Information Science, Yantai University, Yantai, Shandong Province, 264005, P. R. China.

Weihai Zhang is with the College of Electrical Engineering and Automation, Shandong University of Science and Technology, Qingdao, Shandong Province, 266590, P. R. China. (Corresponding author: w_hzhang@163.com).

Manuscript received April 19, 2005; revised August 26, 2015. the random disturbance in the latter is a stationary stochastic process. Since the white noise has infinite bandwidth, some actual systems are more suitable to be modeled as random nonlinear systems from the energy view.

Along with the work of Khas'minskii, Krstić and Deng, Mao and other scholars in stochastic control field, the remarkable development has been achieved (please refer to [5], [8 10] and the references therein). This promotes the study of stochastic nonlinear time-delay systems. [11] designed an output-feedback controller by Lyapunov-based recursive method such that a class of stochastic lower triangular systems with constant delay only appeared in drift term is exponentially stable. For general stochastic nonlinear time-delay systems, [12] proposed some conditions to guarantee that systems solution exists and is unique, and gave the global stability criteria in probability sense. Besides, the adaptive outputfeedback stabilization controller for a class of stochastic lower triangular systems with time-delay is constructed in [12]. By the stability results in [12], [13 constructed an outputfeedback controller to achieve globally asymptotically stable in probability sense for stochastic high-order nonlinear timedelay systems satisfying some assumptions and [14] studied stabilization control for a class of stochastic upper-triangular nonlinear systems with time-delay by state feedback approach. However, for random nonlinear systems, most findings in existing literature (such as [7, 15, 19]) didn't investigate the influence of time-delay phenomenon on systems dynamics behavior. The corresponding results about random nonlinear time-delay systems also have not been reported in existing literature. This motivates us to focus on random nonlinear time-delay systems.

Inspired by [7] and [12], this paper considers the stability of random time-delay systems and regulation control problems for random nonlinear feedback time-delay systems. The main contributions of this paper include three aspects:

(1) For random time-varying delay systems with some general conditions, this paper analyzes the existence and uniqueness of global solution.

(2) Some definitions and criteria on noise-to-state stability (including in the moment sense and in probability sense ) are proposed based on Lyapunov function approach.

(3) Applying the obtained stability results, two classes of regulation control problems are studied by constructing different Lyapunov-Krasovskii functionals, respectively.

This paper is organized as follows. Section II investigates the existence and uniqueness of global solution for random systems with time-delay. Some definitions and noise-to-state stability criteria are proposed in Section III. As applications, 
two kinds of regulation problems are discussed in Section IV and Section V, respectively. In Section VI, two corresponding simulation examples illustrate the feasibility and effectiveness of proposed two controller design procedures. Section VII gives the conclusions of this paper.

Notions: $|x|$ and $x^{T}$ represent the usual Euclidean norm and the transpose of vector $x$, respectively. $\|A\|$ denotes the 2-norm of matrix $\mathrm{A}$ and $\|A\|_{F}$ stands for the Frobenius norm of matrix A. $\mathbb{R}_{+}$and $\mathbb{R}^{n}$ represent the set of nonnegative real numbers and the real $n$-dimensional space, respectively. $C([t-$ $\left.\tau, t] ; \mathbb{R}^{n}\right)$ represents the space of continuous functions $q$ from $[t-\tau, t]$ to $\mathbb{R}^{n}$ with the norm $\left|q_{t}\right|=\sup _{-\tau \leq \theta \leq 0}|q(t+\theta)|$ for $\tau>0$ and $t \geq 0$. The set of functions with continuous $i$-th partial derivative is denoted as $C^{i}$, and the function $W(t, x(t)) \in$ $C^{1,1}\left(\left[t_{0}-\tau, \infty\right) \times \mathbb{R}^{n} ; \mathbb{R}_{+}\right)$means that $W(t, x(t))$ are $C^{1}$ in $t$ and $C^{1}$ in $x . C_{\mathcal{F}_{t_{0}}}^{b}\left(\left[t_{0}-\tau, t_{0}\right] ; \mathbb{R}^{n}\right)$ denotes the family of all $\mathcal{F}_{t_{0}}$-measurable bounded $C\left(\left[t_{0}-\tau, t_{0}\right] ; \mathbb{R}^{n}\right)$-valued random variable $\varphi=\left\{\varphi(\theta): t_{0}-\tau \leq \theta \leq t_{0}\right\}$ with $t_{0} \geq 0 . \gamma(t) \in \mathcal{K}$ means that the function $\gamma(t)$ defined on $\mathbb{R}_{+}$is strictly increasing, continuous and vanish at origin; $\bar{\gamma}(t) \in \mathcal{K}_{\infty}$ implies that $\bar{\gamma}(t) \in \mathcal{K}$ and $\bar{\gamma}(t)$ is unbounded; $\beta(s, t) \in \mathcal{K} \mathcal{L}$ denotes that $\beta(s, t) \in \mathcal{K}$ for each fixed $t$, and $\lim _{t \rightarrow \infty} \beta(s, t)=0$ holds for each fixed $s$. Function $h(s)$ is convex on $D$, if for any $s_{1}, s_{2} \in D, h(s)$ satisfies $h\left(\left(s_{1}+s_{2}\right) / 2\right) \leq\left(h\left(s_{1}\right)+h\left(s_{2}\right)\right) / 2$.

\section{PRELIMINARIES}

In this section, one discusses the existence and uniqueness of solution to random differential delay equations (RDDEs) that are a special class of random functional differential equations (RFDEs). So, one firstly discusses the the existence and uniqueness of solution to RFDEs and then analyze the existence and uniqueness of solution to RDDEs.

\section{A. The existence and uniqueness of solution to RFDEs}

Consider the following random functional differential equation

$$
\dot{x}(t)=f\left(t, x_{t}\right)+g\left(t, x_{t}\right) \xi(t), \quad t \geq t_{0},
$$

with the initial data $x_{t_{0}}=\varphi=\left\{\varphi\left(t_{0}+\theta\right):-\tau \leq \theta \leq 0\right\} \in$ $C_{\mathcal{F}_{t_{0}}}^{b}\left(\left[t_{0}-\tau, t_{0}\right] ; \mathbb{R}^{n}\right)$, where $x_{t}=\{x(t+\theta): \theta \in[-\tau, 0]\}$ is a $C\left([t-\tau, t] ; \mathbb{R}^{n}\right)$-valued stochastic process. The state is $x(t) \in \mathbb{R}^{n}, \xi(t) \in \mathbb{R}^{m}$ represents a piecewise continuous and $\mathcal{F}_{t}$-adapted stochastic process defined on the complete filtered probability space $\left(\Omega, \mathcal{F},\left\{\mathcal{F}_{t}\right\}_{t>t_{0}}, P\right)$, and it has finite $r$-order moment (i.e., $\sup _{t \geq t_{0}} E|\xi(t)|^{r}<K$ with $r \geq 1$ and $K$ being positive constants). For $t_{0} \leq t \leq T<\infty$, functions $f:\left[t_{0}, T\right] \times C\left([t-\tau, t] ; \mathbb{R}^{n}\right) \rightarrow \mathbb{R}^{n}$ and $g$ : $\left[t_{0}, T\right] \times C\left([t-\tau, t] ; \mathbb{R}^{n}\right) \rightarrow \mathbb{R}^{n \times m}$ are Borel measurable, piecewise continuous in $t$ and locally Lipschitz continuous in $x_{t} \in \mathbb{R}^{n}$, moreover, $f(t, 0)$ and $g(t, 0)$ are bounded.

Definition 1: A solution $x(t)$ to equation (1) on $\left[t_{0}-\tau, T\right]$ with initial value $x_{t_{0}}=\varphi$ is an $\mathbb{R}^{n}$-valued stochastic process and satisfies that

(i) $x(t)$ and $\left\{x_{t}\right\}_{t_{0} \leq t \leq T}$ are continuous and $\mathcal{F}_{t}$-adapted.

(ii) $x(t)=x_{t_{0}}+\int_{t_{0}}^{t} f\left(s, x_{s}\right) d s+\int_{t_{0}}^{t} g\left(s, x_{s}\right) \xi(s) d s$ holds almost surely for $t_{0} \leq t \leq T$.

The uniqueness of solution to equation (1) is in almost sure sense, that is to say, a solution $x(t)$ is called to be unique if
$P\left\{x(t)=\bar{x}(t), t_{0}-\tau \leq t \leq T\right\}=1$, where $\bar{x}(t)$ is another solution to equation (1). For any $T>t_{0}-\tau$, if there exists a unique solution to equation (1) on $\left[t_{0}-\tau, T\right]$, equation (1) has a unique solution on $\left[t_{0}-\tau, \infty\right)$.

In order to establish the sufficient conditions, which ensure that the global solution to equation (1) exists and is unique, one introduces the first exit time from a region $U_{k}=\{x$ : $|x|<k\}$ and its limit. Let $\rho_{k}=\inf \left\{t \geq t_{0}:|x(t)| \geq k\right\}$ and $\rho_{\infty}=\lim _{k \rightarrow \infty} \rho_{k}$ almost surely, where $\rho_{k}$ are stopping times, $\inf \phi=\infty$, and $k \geq 1$ is any integer.

Lemma 1: For equation (1), if there is a function $V(t, x(t)) \in C^{1,1}\left(\left[t_{0}-\tau, \infty\right) \times \mathbb{R}^{n} ; \mathbb{R}_{+}\right)$and constants $c_{0}$ and $d_{0}>0$ such that for any integer $k \geq 1$ and $t \geq t_{0}$,

$$
\begin{gathered}
\lim _{k \rightarrow \infty} \inf _{|x| \geq k} V(t, x)=\infty, \\
E V\left(\rho_{k} \wedge t, x\left(\rho_{k} \wedge t\right)\right) \leq d_{0} e^{c_{0} t},
\end{gathered}
$$

then there exists a unique solution on $\left[t_{0}-\tau, \infty\right)$ to equation (11) .

Proof: For every integer $k \geq 1$, one defines the following truncation functions

$$
\begin{aligned}
& f_{k}\left(t, x_{t}\right)=\left\{\begin{array}{lll}
f\left(t, x_{t}\right) & \text { if }\left|x_{t}\right| \leq k \\
f\left(t, k x_{t} /\left|x_{t}\right|\right) & \text { if } & \left|x_{t}\right|>k
\end{array}\right. \\
& g_{k}\left(t, x_{t}\right)=\left\{\begin{array}{lll}
g\left(t, x_{t}\right) & \text { if }\left|x_{t}\right| \leq k \\
g\left(t, k x_{t} /\left|x_{t}\right|\right) & \text { if }\left|x_{t}\right|>k
\end{array}\right.
\end{aligned}
$$

Then $f_{k}\left(t, x_{t}\right)$ and $g_{k}\left(t, x_{t}\right)$ satisfy the Lipschitz condition. Adopting a similar way as Lemma 3 in [7], one can prove that there exists a unique $\mathcal{F}_{t}$-adapted solution $x_{k}(t)$ satisfying

$$
\dot{x}_{k}(t)=f_{k}\left(t, x_{t, k}\right)+g_{k}\left(t, x_{t, k}\right) \xi(t), \quad t \geq t_{0},
$$

with the initial value $x_{t_{0}, k}=\varphi \in C_{\mathcal{F}_{t_{0}}}^{b}\left(\left[t_{0}-\tau, t_{0}\right] ; \mathbb{R}^{n}\right)$, the details of proof is omitted here. It is clear that

$$
x_{k}(t)=x_{k+1}(t), \quad \forall t \in\left[t_{0}, \rho_{k}\right) .
$$

Define $x(t)=x_{k}(t), t \in\left[t_{0}, \rho_{k}\right), k \geq 1$, then it follows from (4) and (5) that for any $t \geq t_{0}$ and $k \geq 1$,

$$
x\left(t \wedge \rho_{k}\right)=\varphi\left(t_{0}\right)+\int_{t_{0}}^{t \wedge \rho_{k}}\left[f\left(s, x_{s, k}\right)+g\left(s, x_{s, k}\right) \xi(s)\right] d s .
$$

First, one considers the case of $\rho_{\infty}<T<\infty$, then

$$
\limsup _{t \rightarrow \rho_{\infty}}|x(t)| \geq \limsup _{k \rightarrow \infty}\left|x\left(\rho_{k}\right)\right|=\limsup _{k \rightarrow \infty}\left|x_{k}\left(\rho_{k}\right)\right|=\infty,
$$

which means that equation (1) has a maximal solution $x(t), t \in$ $\left[t_{0}, \rho_{\infty}\right)$, where $\rho_{\infty}$ is often called the explosion time. The uniqueness of $x_{k}(t)$ on $\left[t_{0}, \rho_{\infty}\right)$ can lead to the uniqueness of the solution $x(t)$ on $\left[t_{0}, \rho_{\infty}\right)$. By now, there exists a unique solution $x(t)$ to equation (1) on $\left[t_{0}-\tau, \rho_{\infty}\right)$ and the solution $x(t)$ is $\mathcal{F}_{t}$-adapted, which can be inferred from the fact that $x_{k}(t)$ is $\mathcal{F}_{t}$-adapted.

Next, one begins to show $\rho_{\infty}=\infty$ almost surely. In fact, if $\rho_{\infty}<\infty$ almost surely, then there exist constants $T>0$ and $\varepsilon>0$ such that $P\left\{\rho_{\infty} \leq T\right\}>\varepsilon$. Because $\lim _{k \rightarrow \infty} \rho_{k}=\rho_{\infty}$ almost surely, there is a integer $k_{0}$ such that

$$
P\left\{\rho_{k} \leq T\right\}>\varepsilon, \quad \forall k \geq k_{0} .
$$


For any fixed $k\left(k \geq k_{0}\right)$, let $t=T$ in (3), then one has $E V\left(T \wedge \rho_{k}, x\left(T \wedge \rho_{k}\right)\right) \leq d_{0} e^{c_{0} T}$, from which one also can obtain

$$
E\left[V\left(\rho_{k}, x\left(\rho_{k}\right)\right) I_{\left\{\rho_{k} \leq T\right\}}\right] \leq d_{0} e^{c_{0} T} .
$$

On the other hand, one defines $\varrho_{k, T}=\inf \{V(t, x(t))$ : $\left.|x(t)| \geq k, t \in\left[t_{0}, T\right]\right\}$, then $\lim _{k \rightarrow \infty} \varrho_{k, T}=\infty$ by (2). It follow from (6) and (7) that $d_{0} e^{c_{0} T} \geq \varrho_{k, T} P\left\{\rho_{k} \leq T\right\}>$ $\varepsilon \varrho_{k, T}$. Since letting $k \rightarrow \infty$ yields a contradiction, one has $\rho_{\infty}=\infty$ almost surely. So, there is a unique global solution $x(t)$ on $\left[t_{0}-\tau, \infty\right)$ for equation (11).

Lemma 2: For equation (1), aussume that there are a function $V(t, x(t)) \in C^{1,1}\left(\left[t_{0}-\tau, \infty\right) \times \mathbb{R}^{n} ; \mathbb{R}_{+}\right)$and a constant $K_{1}>0$ such that

$$
\begin{gathered}
\lim _{|x| \rightarrow \infty} \inf _{t \geq t_{0}} V(t, x)=\infty, \\
E \dot{V}(t, x) \leq K_{1}(1+E V(t, x)+E V(t+\theta, x(t+\theta))),
\end{gathered}
$$

where $-\tau \leq \theta \leq 0$ and $t \geq t_{0}$, then equation (11) has a unique solution on $\left[t_{0}-\tau, \infty\right)$.

Proof: From the proof of Lemma 1 one knows that there is a unique maximal local solution $x(t)$ on $\left[t_{0}-\tau, \rho_{\infty}\right)$ for equation (11). Therefore, one need to testify that $\rho_{\infty}=\infty$ almost surely. For any $k \geq 1$, let $\varrho_{k}=\rho_{\infty} \wedge \inf \left\{t_{0} \leq t<\rho_{\infty}:|x(t)| \geq k\right\}$. It is certain that $\varrho_{\infty} \leq \rho_{\infty}$ and $\varrho_{\infty}=\lim _{k \rightarrow \infty} \varrho_{k}$ almost surely.

By Fubini's theorem [Theorem 2.39, 20] and (9), one has

$$
\begin{aligned}
& E V\left(t \wedge \varrho_{k}, x\left(t \wedge \varrho_{k}\right)\right) \\
\leq & \bar{H}(t)+K_{1} \int_{t_{0}}^{t} \sup _{t_{0} \leq v \leq s}\left[E V\left(v \wedge \varrho_{k}, x\left(v \wedge \varrho_{k}\right)\right)\right] d s \\
\triangleq & H^{*}(t) .
\end{aligned}
$$

where $t \geq t_{0}, k \geq 1$, and $\bar{H}(t)=E V\left(t_{0}, x\left(t_{0}\right)\right)+K_{1}(t-$ $\left.t_{0}\right)+K_{1} \int_{t_{0}}^{t} E V(s+\theta, x(s+\theta)) d s>0$. Because $H^{*}(t)$ is a increasing function of $t$, one can obtain the following inequality from 10

$$
\begin{aligned}
& \sup _{t_{0} \leq v \leq t}\left[E V\left(v \wedge \varrho_{k}, x\left(v \wedge \varrho_{k}\right)\right)\right] \\
& \leq \bar{H}(t)+K_{1} \int_{t_{0}}^{t} \sup _{t_{0} \leq v \leq s}\left[E V\left(v \wedge \varrho_{k}, x\left(v \wedge \varrho_{k}\right)\right)\right] d s .
\end{aligned}
$$

By Gronwall's inequality [Theorem 1.20, 20], one gets

$$
\sup _{t_{0} \leq v \leq t}\left[E V\left(v \wedge \varrho_{k}, x\left(v \wedge \varrho_{k}\right)\right)\right] \leq \bar{H}(t) e^{K_{1}\left(t-t_{0}\right)} .
$$

It is certain that

$$
E V\left(t \wedge \varrho_{k}, x\left(t \wedge \varrho_{k}\right)\right) \leq \bar{H}(t) e^{K_{1} t}, \quad \forall t \geq t_{0} .
$$

On the other hand, for any $R \geq 0$, one defines that $\delta(R)=\inf _{R<|x|, t_{0}<t} V(t, x(t))$, then it is easy to show that $\delta(|x(t)|) \leq V(t, x(\bar{t}))$ and $\lim _{R \rightarrow \infty} \delta(R)=\infty$ which comes from (8). It follows from (11) that

$$
P\left\{\varrho_{k} \leq t\right\} \leq \frac{E \delta\left(\left|x\left(t \wedge \varrho_{k}\right)\right|\right)}{\delta(k)} \leq \frac{\bar{H}(t) e^{K_{1} t}}{\delta(k)} .
$$

Letting first $k \rightarrow \infty$ and then $t \rightarrow \infty, P\left\{\varrho_{\infty}<\infty\right\}=0$ can be obtained, that is, $\varrho_{\infty}=\infty$ almost surely. This means that $\rho_{\infty}=\infty$ almost surely. Thus, equation (1) has a unique solution on $\left[t_{0}-\tau, \infty\right)$.

\section{$B$. The existence and uniqueness of solution to RDDEs}

For the following random differential delay equations

$$
\dot{x}=f(t, x(t-\tau(t)), x)+g(t, x(t-\tau(t)), x) \xi(t),
$$

with the initial value $x_{t_{0}}=\varphi=\left\{\varphi(t): t_{0}-\tau \leq t \leq t_{0}\right\} \in$ $C_{\mathcal{F}_{t_{0}}}^{b}\left(\left[t_{0}-\tau, t_{0}\right] ; \mathbb{R}^{n}\right)$. System state is $x(t) \in \mathbb{R}^{n}$, and the random disturbance $\xi(t) \in \mathbb{R}^{m}$ is in accord with that of system (11). Borel measurable function $\tau(t)$ is defined on $\left[t_{0}, \infty\right)$ and takes values on $[0, \tau]$. Borel measurable functions $f:\left[t_{0}, \infty\right) \times$ $\mathbb{R}^{n} \times \mathbb{R}^{n} \rightarrow \mathbb{R}^{n}$ and $g:\left[t_{0}, \infty\right) \times \mathbb{R}^{n} \times \mathbb{R}^{n} \rightarrow \mathbb{R}^{n \times m}$ satisfy locally Lipschitz condition with respect to $x(t-\tau(t))$ and $x(t)$, respectively. In addition, $f(t, 0,0)$ and $g(t, 0,0)$ are bounded.

One introduces $F\left(t, \bar{x}_{t}\right)=f\left(t, \bar{x}_{t}(-\tau(t)), \bar{x}_{t}(0)\right)=$ $f(t, x(t-\tau(t)), x(t))$ and $G\left(t, \bar{x}_{t}\right)=g\left(t, \bar{x}_{t}(-\tau(t)), \bar{x}_{t}(0)\right)=$ $g(t, x(t-\tau(t)), x(t))$, then equation (12) can be rewritten as

$$
\dot{x}=F\left(t, \bar{x}_{t}\right)+G\left(t, \bar{x}_{t}\right) \xi(t), \quad t \geq t_{0} .
$$

This implies that RDDEs are in fact a special class of RFDEs. Therefore, one can obtain the following sufficient conditions such that system (12) has a unique global solution.

Lemma 3: For system (12), if there are a function $V(t, x(t)) \in C^{1,1}\left(\left[t_{0}-\tau, \infty\right) \times \mathbb{R}^{n} ; \mathbb{R}_{+}\right)$and constants $c_{0}$ , $d_{0}>0$ such that (2) and (3) hold for any integer $k \geq 1$ and $t \geq t_{0}$, then there is a unique solution to system (12) with initial value $x_{t_{0}}=\varphi \in C_{\mathcal{F}_{t_{0}}}^{b}\left(\left[t_{0}-\tau, t_{0}\right] ; \mathbb{R}^{n}\right)$.

Proof: For system (12), since both $f(t, x(t-\tau(t)), x)$ and $g(t, x(t-\tau(t)), x)$ are Borel measurable functions and satisfy locally Lipschitz condition with respect to $x(t-\tau(t))$ and $x$, respectively. In other words, for any $R>0$, there is a positive constant $L_{R}$ such that for $\forall x_{1}, x_{2} \in U_{R}, x_{1} \neq x_{2}$ and $\forall x_{1}(t-\tau(t)), x_{2}(t-\tau(t)) \in U_{R}, x_{1}(t-\tau(t)) \neq x_{2}(t-\tau(t))$,

$$
\begin{aligned}
& \left|f\left(t, x_{1}(t-\tau(t)), x_{1}\right)-f\left(t, x_{2}(t-\tau(t)), x_{2}\right)\right| \\
\leq & L_{R}\left(\left|x_{1}-x_{2}\right|+\left|x_{1}(t-\tau(t))-x_{2}(t-\tau(t))\right|\right), \\
& || g\left(t, x_{1}(t-\tau(t)), x_{1}\right)-g\left(t, x_{2}(t-\tau(t)), x_{2}\right)|| \\
\leq & L_{R}\left(\left|x_{1}-x_{2}\right|+\left|x_{1}(t-\tau(t))-x_{2}(t-\tau(t))\right|\right) .
\end{aligned}
$$

Note that $\left|q_{t}\right|=\sup _{0 \leq \tau(t) \leq \tau}|q(t-\tau(t))|$, then one has

$$
\begin{aligned}
& \left|x_{1}-x_{2}\right| \vee\left|x_{1}(t-\tau(t))-x_{2}(t-\tau(t))\right| \\
\leq & \left|\bar{x}_{1, t}-\bar{x}_{2, t}\right| .
\end{aligned}
$$

It follows from (14) and (15) that

$$
\begin{aligned}
& \left|F\left(t, \bar{x}_{1, t}\right)-F\left(t, \bar{x}_{2, t}\right)\right| \vee\left\|G\left(t, \bar{x}_{1, t}\right)-G\left(t, \bar{x}_{2, t}\right)\right\| \\
\leq & 2 L_{R}\left|\bar{x}_{1, t}-\bar{x}_{2, t}\right| .
\end{aligned}
$$

This means that equation (13) satisfies the locally Lipschitz condition. From Lemma 11 there is a unique solution on $\left[t_{0}-\tau, \infty\right)$ to system (13) if there are a positive function $V(t, x(t)) \in C^{1,1}\left(\left[t_{0}-\tau, \infty\right) \times \mathbb{R}^{n} ; \mathbb{R}_{+}\right)$, constant $c_{0}$ and positive constant $d_{0}$ satisfying (2) and (3). Since equation (12) is equivalent to equation (13), there exists a unique solution to system (12).

Definition 2: [Definition 1, 7] For any $\varepsilon>0$, if there is a positive constant $\epsilon$ such that $P\left\{\sup _{t \geq t_{0}}|\phi(t)|>\epsilon\right\} \leq \varepsilon$ holds, then the stochastic process $\phi(t)$ is bounded in probability.

Lemma 4: For system [12), if there are a function $V(t, x(t)) \in C^{1,1}\left(\left[t_{0}-\tau, \infty\right) \times \mathbb{R}^{n} ; \mathbb{R}_{+}\right)$and a constant $K_{2}>$ 
0 such that for any $t \geq t_{0}, \lim _{|x| \rightarrow \infty} \inf _{t>t_{0}} V(t, x)=\infty$ and $E \dot{V}(t, x) \leq K_{2}(1+E V(t, x)+E V(t-\tau(t), x(t-\tau(t)))$ hold, then system (12) has a unique solution.

Remark 1: Since the proof of Lemma 4 is similar to that of Lemma 2, the detailed proof of Lemma 4 is omitted here.

Lemma 5: For system (12), if there exist a function $V(t, x(t)) \in C^{1,1}\left(\left[t_{0}-\tau, \infty\right) \times \mathbb{R}^{n} ; \mathbb{R}_{+}\right)$and a positive constant $d_{0}$ such that for any integer $k \geq 1$, (2) and

$$
E V\left(t \wedge \rho_{k}, x\left(t \wedge \rho_{k}\right)\right) \leq d_{0}
$$

hold, then system (12) has a unique solution and this unique solution is bounded in probability.

Proof: According to Lemma 3, system (12) has a unique global solution. From 17, one has

$$
E V(t, x(t))=\lim _{k \rightarrow \infty} E V\left(\rho_{k} \wedge t, x\left(\rho_{k} \wedge t\right)\right) \leq d_{0} .
$$

On the other hand, by Lemma 1.4 in $[5]$, one gets

$$
\begin{aligned}
E V(t, x(t)) & \geq \int_{\left\{\sup _{t \geq t_{0}}|x(t)|>r\right\}} V(t, x(t)) d P \\
& \geq P\left\{\sup _{t \geq t_{0}}|x(t)|>r\right\} \inf _{t \geq t_{0},|x(t)|>r} V(t, x(t)) .
\end{aligned}
$$

Thus, there is a positive constant $r$ such that for any $\varepsilon>0$,

$$
P\left\{\sup _{t \geq t_{0}}|x(t)|>r\right\} \leq \frac{d_{0}}{\inf _{t \geq t_{0},|x(t)|>r} V(t, x(t))}<\varepsilon .
$$

This implies that the unique global solution of system (12) is bounded in probability.

Lemma 6: 21] $y(t), k(t)$ and $w(t)$ are continuous functions on $\left[t_{0}, \infty\right)$ such that

$$
\limsup _{\Delta t \rightarrow 0^{+}} \frac{y(t+\Delta t)-y(t)}{\Delta t}=D^{+} y(t) \leq k(t) y(t)+w(t)
$$

holds for almost all $t \in\left[t_{0}, \infty\right)$. Then,

$$
y(t) \leq y\left(t_{0}\right) e^{\int_{t_{0}}^{t} k(s) d s}+\int_{t_{0}}^{t} e^{\int_{s}^{t} k(v) d v} w(s) d s, \quad \forall t \geq t_{0} .
$$

Lemma 7: 22 For functions $\chi_{1}, \chi_{2}, \cdots, \chi_{n}$ and $l \geq 1$, the following inequality holds

$$
\left|\chi_{1}+\chi_{2}+\cdots+\chi_{n}\right|^{l} \leq n^{l-1}\left(\left|\chi_{1}\right|^{l}+\left|\chi_{2}\right|^{l}+\cdots+\left|\chi_{n}\right|^{l}\right) \text {. }
$$

\section{MAIN RESULTS}

In this section, one discusses the noise-to-state stability of random time-delay systems (12) in the moment sense and in probability sense, respectively. First, for random nonlinear time-delay systems (12), one gives some definitions about the ultimate boundedness of system state and noise-to-state stability.

Definition 3: If there exist functions $\gamma \in \mathcal{K}$ and $\beta \in \mathcal{K} \mathcal{L}$ such that $E|x(t)|^{m} \leq \beta\left(|\varphi|, t-t_{0}\right)+\gamma\left(\sup _{t_{0} \leq l \leq t} E|\xi(l)|^{r}\right)$ holds for system (12) with any given initial value $\varphi \in$ $C_{\mathcal{F}_{t_{0}}}^{b}\left(\left[t_{0}-\tau, t_{0}\right] ; \mathbb{R}^{n}\right)$, where $m$ is a positive constant and $|\varphi|=\sup _{t_{0}-\tau \leq s \leq t_{0}}|\varphi(s)|$, then system (12) is called to be noise-to-state stable in the $\mathbf{m}$-th moment sense(NSS-m-M).

Definition 4: For any $\varepsilon>0$, if there exist functions $\gamma \in \mathcal{K}$ and $\beta \in \mathcal{K} \mathcal{L}$ such that $P\left\{|x(t)| \leq \beta\left(|\varphi|, t-t_{0}\right)+\right.$ $\left.\gamma\left(\sup _{t_{0} \leq l \leq t} E|\xi(l)|^{r}\right)\right\} \geq 1-\varepsilon$ holds for system with any given initial value $\varphi \in C_{\mathcal{F}_{t_{0}}}^{b}\left(\left[t_{0}-\tau, t_{0}\right] ; \mathbb{R}^{n}\right)$, where $|\varphi|=\sup _{t_{0}-\tau \leq s \leq t_{0}}|\varphi(s)|$, then system (12) is called to be noise-to-state stable in probability sense (NSS-P).

Definition 5: Let $m>0$ be a constant. If there is a function $\gamma \in \mathcal{K}$ such that $\lim _{t \rightarrow \infty} E|x(t)|^{m} \leq \gamma(K)$ holds, then the state $x(t)$ of system (12) is ultimately bounded in the $\mathbf{m}$-th moment sense (UB-m-M).

Definition 6: For any $\varepsilon>0$, if there is a function $\gamma \in \mathcal{K}$ such that $P\left\{\lim _{t \rightarrow \infty}|x(t)|>\gamma(K)\right\}<\varepsilon$ holds, then the state $x(t)$ of system (12) is ultimately bounded in probability sense (UB-P).

Next, one addresses the criteria on noise-to-state stability of random time-delay systems in the moment sense and in probability sense, respectively.

Theorem 1: For system (12), if there are a function $V(t, x) \in C^{1,1}\left(\left[t_{0}-\tau, \infty\right) \times \mathbb{R}^{n} ; \mathbb{R}_{+}\right)$, positive constants $m, c_{1}, c_{2}, c_{3}, c$ and non-negative constant $d_{c}$ such that

$$
\begin{gathered}
c_{1}|x(t)|^{m} \leq V(t, x) \leq c_{2}\left(\sup _{-\tau \leq s \leq 0}|x(t+s)|\right)^{m}, \\
\dot{V}(t, x) \leq-c V(t, x)+c_{3}|\xi(t)|^{r}+d_{c},
\end{gathered}
$$

then (i) system (12) has a unique solution on $\left[t_{0}-\tau, \infty\right)$; (ii) system (12) is NSS-m-M; (iii) the state of system (12) is UB-m-M.

Proof: From (18), it yields that

$$
\lim _{|x| \rightarrow \infty} \inf _{t \geq t_{0}} V(t, x)=\infty .
$$

Taking expectations on both sides of (19), then

$$
E \dot{V}(t, x) \leq-c E V(t, x)+c_{3} E|\xi(t)|^{r}+d_{c} .
$$

According to Lemma 4 it follows from (20) and 21) that system (12) has a unique global solution.

Letting $\nu(t)=E V(t, x)$ and using Fubini's theorem [Theorem 2.39, 20, then 21 can be turned into

$\nu(t+\varepsilon) \leq \nu(t)+\int_{t}^{t+\varepsilon}\left(-c \nu(s)+c_{3} K+d_{c}\right) d s, \forall \varepsilon>0, t \geq t_{0}$.

So,

$$
D^{+} \nu(t) \leq-c \nu(t)+c_{3} K+d_{c} .
$$

By Lemma 6, one arrives at

$$
\begin{aligned}
\nu(t) & \leq \nu\left(t_{0}\right) e^{-c\left(t-t_{0}\right)}+\int_{t_{0}}^{t} e^{-c(t-s)}\left(c_{3} K+d_{c}\right) d s \\
& \leq \nu\left(t_{0}\right) e^{-c\left(t-t_{0}\right)}+\frac{c_{3} K+d_{c}}{c}-\frac{c_{3} K+d_{c}}{c} e^{c\left(t_{0}-t\right)} \\
& \leq \nu\left(t_{0}\right) e^{-c\left(t-t_{0}\right)}+\frac{c_{3} K+d_{c}}{c}
\end{aligned}
$$

together with 18, one can deduce that

$$
E|x(t)|^{m} \leq \frac{c_{2}}{c_{1}}|\varphi|^{m} e^{-c\left(t-t_{0}\right)}+\frac{c_{3} K+d_{c}}{c c_{1}} .
$$

Thus, the system (12) is NSS-m-M.

In addition, letting $t \rightarrow \infty$ in (22), it yields that

$$
\lim _{t \rightarrow \infty} E|x(t)|^{m} \leq \frac{c_{3} K+d_{c}}{c c_{1}} .
$$

This means that the state of system (12) is UB-m-M. 
Theorem 2: For system (12), if there are a function $V(x) \in$ $C\left(\mathbb{R}^{n} ; \mathbb{R}_{+}\right)$, functions $\gamma \in \mathcal{K}, \gamma_{1} \in \mathcal{K} \mathcal{L}, \gamma_{2} \in \mathcal{K} \mathcal{L}$ and positive constant $a_{0}$ such that

$$
\begin{aligned}
& \gamma_{1}(|x(t)|) \leq V(x) \leq \gamma_{2}\left(\sup _{-\tau \leq s \leq 0}|x(t+s)|\right), \\
& \dot{V}(x) \leq-\gamma\left(\sup _{-\tau \leq s \leq 0}|x(t+s)|\right)+a_{0}|\xi(t)|^{r},
\end{aligned}
$$

then system (12) has a unique solution. If $\gamma \circ \gamma_{2}^{-1}(\cdot)$ is a convex function, then system (12) is NSS-P and the state of system (12) is UB-P.

Proof: From (23) and 24), one obtains (20) and

$$
\begin{aligned}
E \dot{V}(x) & \leq-E\left[\gamma\left(\sup _{-\tau \leq s \leq 0}|x(t+s)|\right)\right]+a_{0} E|\xi(t)|^{r} \\
& \leq a_{0} K(1+E V(x)+E V(x(t-\tau(t))),
\end{aligned}
$$

respectively. So system (12) has a unique solution in the light of Lemma 4

If $\gamma \circ \gamma_{2}^{-1}(\cdot)$ is a convex function, then by (25) and Jensen's inequality [22], and let $\bar{\nu}(t)=E V(x)$, one gets

$$
\begin{aligned}
\dot{\bar{\nu}}(t) & \leq-E\left[\gamma\left(\sup _{-\tau \leq s \leq 0}|x(t+s)|\right)\right]+a_{0} E|\xi(t)|^{r} \\
& \leq-\gamma \circ \gamma_{2}^{-1}(\bar{\nu}(t))+a_{0} \sup _{t_{0} \leq l \leq t} E|\xi(l)|^{r} .
\end{aligned}
$$

Following the thread of Theorem 2 of [7], there exist functions $\tilde{\beta} \in \mathcal{K} \mathcal{L}$ and $\tilde{\gamma} \in \mathcal{K}$ such that $P\left\{|x(t)|>\tilde{\beta}\left(|\varphi|, t-t_{0}\right)+\right.$ $\left.\tilde{\gamma}\left(a_{0} \sup _{t_{0} \leq l \leq t} E|\xi(l)|^{r}\right)\right\}<\varepsilon$ holds, and letting $t \rightarrow \infty$, then one has

$$
P\left\{\lim _{t \rightarrow \infty}|x(t)|>\tilde{\gamma}\left(a_{0} K\right)\right\}<\varepsilon .
$$

Thus, system (12) is NSS-P and the system state is UB-P.

Remark 2: (i) In Theorem 11 if 19) is replaced by $\dot{V}(t, x) \leq$ $-c\left(\sup _{-\tau \leq s \leq 0}|x(t+s)|\right)^{m}+c_{3}|\xi(t)|^{r}+d_{c}$, the result remains valid. In fact, by (18), one has

$$
\begin{aligned}
\dot{V}(t, x) & \leq-c\left(\sup _{-\tau \leq s \leq 0}|x(t+s)|\right)^{m}+c_{3}|\xi(t)|^{r}+d_{c} \\
& \leq-\frac{c}{c_{2}} V(t, x)+c_{3}|\xi(t)|^{r}+d_{c} .
\end{aligned}
$$

(ii) From (25), it is certain that if (24) is replaced by $\dot{V}(x) \leq$ $-\gamma \circ \gamma_{2}^{-1}(V(x))+a_{0}|\xi(t)|^{r}$, Theorem 2 still holds for system (12).

Remark 3: The random disturbance $\xi(t)$ in RDDE (12) satisfies the assumption $\sup _{t \geq t_{0}} E|\xi(t)|^{r}<K$, under which one gives the criteria on noise-to-state stability of RDDE (12). If the random disturbance $\xi(t)$ in RDDE (12) satisfies that

$$
E|\xi(t)|^{r} \leq \bar{d}_{0} e^{\bar{c}_{0} t}, \quad t \geq t_{0},
$$

where $\bar{c}_{0}$ is a constant and $\bar{d}_{0}$ is a positive constant, then by adopting the similar way used in section III of [7], one knows that the result (i) and (ii) in Theorem 1 still hold, but the result (iii) does not necessarily hold. Similarly, If (23) and (24) hold in Theorem 2 for RDDE (12) satisfying (26), then system (12) has a unique solution on $\left[t_{0}-\tau, \infty\right)$. Furthermore, system (12] is also NSS-P if $\gamma \circ \gamma_{2}^{-1}(\cdot)$ is a convex function, but the state of system (12) is not necessarily UB-P.

\section{APPLICATION 1: STATE FEEDBACK REGULATION CONTROL}

Consider the following random nonlinear strict-feedback system with time-varying delay

$$
\left\{\begin{array}{c}
\dot{x}_{1}(t)=x_{2}(t)+f_{1}+g_{1} \xi_{1}(t), \\
\dot{x}_{2}(t)=x_{3}(t)+f_{2}+g_{2} \xi_{2}(t), \\
\vdots \\
\dot{x}_{n}(t)=u(t)+f_{n}+g_{n} \xi_{n}(t), \\
y(t)=x_{1}(t) .
\end{array}\right.
$$

where $f_{i}=f_{i}\left(t, \bar{x}_{i}(t-\tau(t)), \bar{x}_{i}(t)\right), g_{i}=g_{i}\left(t, \bar{x}_{i}(t-\right.$ $\left.\tau(t)), \bar{x}_{i}(t)\right), \bar{x}_{i}(t-\tau(t))=\left[x_{1}(t-\tau(t)), \cdots, x_{i}(t-\tau(t))\right]^{T}$ and $\bar{x}_{i}(t)=\left[x_{1}(t), \cdots, x_{i}(t)\right]^{T}$. System state is $x(t)=$ $\left[x_{1}(t), x_{2}(t), \cdots, x_{n}(t)\right]^{T} \in \mathbb{R}^{n}$ with the initial value $x_{t_{0}}=$ $\varphi \in C_{\mathcal{F}_{t_{0}}}^{b}\left(\left[t_{0}-\tau, t_{0}\right] ; \mathbb{R}^{n}\right)$, and system output $y(t) \in \mathbb{R}$ and system input $u(t) \in \mathbb{R}$. Borel measurable function $\tau(t):\left[t_{0}, \infty\right) \rightarrow[0, \tau]$ denotes time-varying delay and satisfies $\dot{\tau}(t) \leq \tau^{*}<1$, where $\tau$ and $\tau^{*}$ are known constants. Stochastic process $\xi(t)=\left[\xi_{1}(t), \xi_{2}(t), \cdots, \xi_{n}(t)\right]^{T} \in \mathbb{R}^{n}$ defined on the complete filtered probability space $\left(\Omega, \mathcal{F},\left\{\mathcal{F}_{t}\right\}_{t>t_{0}}, P\right)$ is $\mathcal{F}_{t}$-adapted and piecewise continuous. $f_{i}, g_{i}, i=1,2, \cdots, n$ are locally Lipschitz continuous functions in $\bar{x}_{i}(t-\tau(t))$ and $\bar{x}_{i}(t)$, respectively. $f(t, 0,0)$ and $g(t, 0,0)$ are bounded.

For system [27), one proposes the following assumptions: H0: The random disturbance $\xi(t)$ satisfies

$$
\sup _{t \geq t_{0}} E|\xi(t)|^{4}<K .
$$

H1: The system state $x(t)=\left[x_{1}(t), \cdots, x_{n}(t)\right]^{T}$ is available. H2: There exists a positive constant $\bar{\theta}$ such that for every $i \in\{1,2, \cdots, n\}$,

$$
\begin{aligned}
\left|f_{i}\left(t, \bar{x}_{i}(t-\tau(t)), \bar{x}_{i}(t)\right)\right| & \leq \bar{\theta} \sum_{j=1}^{i}\left(\left|x_{j}(t-\tau(t))\right|+\left|x_{j}(t)\right|\right), \\
\left|g_{i}\left(t, \bar{x}_{i}(t-\tau(t)), \bar{x}_{i}(t)\right)\right|^{2} & \leq \bar{\theta} \sum_{j=1}^{i}\left(\left|x_{j}(t-\tau(t))\right|+\left|x_{j}(t)\right|\right) .
\end{aligned}
$$

The aim in this section is to design a controller by state feedback method such that the output $y(t)$ can be asymptotically regulated to a neighborhood of the zero that is arbitrarily small and the system state is ultimately bounded in the meansquare sense.

First, introducing the transformations

$$
z_{1}(t)=y(t), z_{i+1}(t)=x_{i+1}(t)-\alpha_{i}\left(y(t), \bar{x}_{i-1}(t)\right),
$$

where $i=1,2, \cdots, n$ and $x_{n+1}(t)=u(t), z_{n+1}(t)=0$, $\bar{x}_{0}(t)=0$, and stabilizing functions $\alpha_{i}(i=1, \cdots, n)$ are to be constructed.

Next, one constructs the Lyapunov-Krasovskii functional step by step.

Step 1 Choosing

$$
V_{1}=V_{\tau 1}+\frac{1}{2} z_{1}^{2}, \quad V_{\tau 1}=n \int_{t-\tau(t)}^{t} e^{s-t} z_{1}^{2}(s) d s,
$$


and noting that $\dot{\tau}(t) \leq \tau^{*}<1$ and $0 \leq \tau(t) \leq \tau$, the derivative of $V_{1}$ satisfies

$$
\begin{aligned}
\dot{V}_{1} \leq & z_{1}\left(\alpha_{1}+z_{2}+f_{1}+g_{1} \xi_{1}\right)+n z_{1}^{2} \\
& -n e^{-\tau}\left(1-\tau^{*}\right) z_{1}^{2}(t-\tau(t))-V_{\tau 1} .
\end{aligned}
$$

Applying Young's inequality and (28), one gets

$$
\begin{aligned}
z_{1} z_{2} & \leq \frac{1}{2} z_{1}^{2}+\frac{1}{2} z_{2}^{2}, \\
z_{1} f_{1} & \leq \Omega_{1} z_{1}^{2}+\frac{\left(1-\tau^{*}\right) e^{-\tau}}{2} z_{1}^{2}(t-\tau(t)), \\
z_{1} g_{1} \xi_{1} & \leq \Lambda_{1} z_{1}^{2}+\frac{\left(1-\tau^{*}\right) e^{-\tau}}{2} z_{1}^{2}(t-\tau(t))+\pi_{1}\left|\xi_{1}\right|^{4},
\end{aligned}
$$

where $\Omega_{1}=\bar{\theta}+\frac{1}{2\left(1-\tau^{*}\right)} \bar{\theta}^{2} e^{\tau}, \Lambda_{1}=\frac{1}{2} \bar{\theta}+\frac{1}{8\left(1-\tau^{*}\right)} \bar{\theta}^{2} e^{\tau}+\frac{1}{16 \pi_{1}}$, and $\pi_{1}$ is a positive design parameter. Substituting (30)-(32) into 29] yields

$$
\begin{gathered}
\dot{V}_{1} \leq z_{1}\left(n z_{1}+\Omega_{1} z_{1}+\Lambda_{1} z_{1}+\frac{1}{2} z_{1}+\alpha_{1}\right)+\frac{1}{2} z_{2}^{2}+\pi_{1}\left|\xi_{1}\right|^{4} \\
-(n-1) e^{-\tau}\left(1-\tau^{*}\right) z_{1}^{2}(t-\tau(t))-V_{\tau 1} .
\end{gathered}
$$

Selecting the stabilizing function $\alpha_{1}=-2 n z_{1}-\Omega_{1} z_{1}-\Lambda_{1} z_{1}-$ $\frac{1}{2} z_{1} \triangleq-\beta_{1} z_{1}$, then one has

$$
\begin{aligned}
\dot{V}_{1} \leq & \frac{1}{2} z_{2}^{2}+\pi_{1}\left|\xi_{1}\right|^{4}-n z_{1}^{2}-V_{\tau 1} \\
& -(n-1)\left(1-\tau^{*}\right) e^{-\tau} z_{1}^{2}(t-\tau(t)) .
\end{aligned}
$$

Step i $(i=2,3, \cdots, n)$ Suppose that $V_{i-1}=V_{i-2}+$ $\frac{1}{2} z_{i-1}^{2}+(n-i+2) \int_{t-\tau(t)}^{t} e^{s-t} z_{i-1}^{2}(s) d s$ and a series of stabilizing functions $\alpha_{j}(j=1,2, \cdots, i-1)$ have been constructed such that

$$
\begin{aligned}
\dot{V}_{i-1} \leq & \frac{1}{2} z_{i}^{2}+\sum_{j=1}^{i-1} \pi_{j}\left|\xi_{j}\right|^{4}+\sum_{j=1}^{i-2} \sum_{l=j+1}^{i-1} \pi_{l j}\left|\xi_{j}\right|^{4} \\
& -(n-i+1) e^{-\tau}\left(1-\tau^{*}\right) \sum_{j=1}^{i-1} z_{j}^{2}(t-\tau(t)) \\
& -(n-i+2) \sum_{j=1}^{i-1} z_{j}^{2}-\sum_{j=1}^{i-1} V_{\tau j}
\end{aligned}
$$

where $V_{\tau j}=(n-j+1) \int_{t-\tau(t)}^{t} e^{s-t} z_{j}^{2}(s) d s, j=1, \cdots, i-1$. In what follows, one will prove that (33) also holds for $V_{i}$.

Let $V_{i}=V_{i-1}+\frac{1}{2} z_{i}^{2}+(n-i+1) \int_{t-\tau(t)}^{t} e^{s-t} z_{i}^{2}(s) d s$, then

$$
\begin{aligned}
\dot{V}_{i} \leq \dot{V}_{i-1}+z_{i}\left(z_{i+1}+\alpha_{i}+f_{i}+g_{i} \xi_{i}\right)+(n-i+1) z_{i}^{2} \\
-z_{i} \sum_{j=1}^{i-1} \frac{\partial \alpha_{i-1}}{\partial x_{j}}\left(x_{j+1}+f_{j}+g_{j} \xi_{j}\right)-V_{\tau i} \\
-(n-i+1) e^{-\tau}\left(1-\tau^{*}\right) z_{i}^{2}(t-\tau(t))
\end{aligned}
$$

where

$$
-\frac{\partial \alpha_{i-1}}{\partial x_{j}}=\prod_{k=j}^{i-1} \beta_{k} \triangleq \Xi_{j(i-1)}, \quad j=1,2, \cdots i-1 .
$$

By H2, 35 and Young's inequality, one obtains

$$
z_{i} z_{i+1} \leq \frac{1}{2} z_{i}^{2}+\frac{1}{2} z_{i+1}^{2},
$$

$$
\begin{aligned}
& z_{i} f_{i}-z_{i} \sum_{j=1}^{i-1} \frac{\partial \alpha_{i-1}}{\partial x_{j}}\left(x_{j+1}+f_{j}\right) \\
\leq & \sum_{l=1}^{i-1} \frac{1}{2} z_{l}^{2}+\Omega_{i} z_{i}^{2}+\frac{e^{-\tau}\left(1-\tau^{*}\right)}{2} \sum_{l=1}^{i} z_{l}^{2}(t-\tau(t)), \\
& z_{i} g_{i} \xi_{i}-z_{i} \sum_{j=1}^{i-1} \frac{\partial \alpha_{i-1}}{\partial x_{j}} g_{j} \xi_{j} \\
\leq & \sum_{l=1}^{i-1} \frac{1}{2} z_{l}^{2}+\Lambda_{i} z_{i}^{2}+\frac{e^{-\tau}\left(1-\tau^{*}\right)}{2} \sum_{l=1}^{i} z_{l}^{2}(t-\tau(t)) \\
& +\pi_{i}\left|\xi_{i}\right|^{4}+\sum_{j=1}^{i-1} \pi_{i j}\left|\xi_{j}\right|^{4}
\end{aligned}
$$

where $\pi_{i}$ and $\pi_{i j}$ are positive design parameters. The detail proofs of (37) and (38) are given in Appendix A and Appendix B, respectively. Substituting (33), 36)-(38) to (34) leads

$$
\begin{aligned}
\dot{V}_{i} \leq & z_{i}\left(z_{i}+\alpha_{i}+2(n-i+1) z_{i}+\Omega_{i} z_{i}+\Lambda_{i} z_{i}\right) \\
& +\frac{1}{2} z_{i+1}^{2}+\sum_{j=1}^{i} \pi_{j}\left|\xi_{j}\right|^{4}+\sum_{j=1}^{i-1} \sum_{l=j+1}^{i} \pi_{l j}\left|\xi_{j}\right|^{4} \\
& -(n-i+1) \sum_{j=1}^{i} z_{j}^{2}-\sum_{j=1}^{i} V_{\tau j} \\
& -(n-i) e^{-\tau}\left(1-\tau^{*}\right) \sum_{j=1}^{i} z_{j}^{2}(t-\tau(t)) .
\end{aligned}
$$

Choosing the stabilizing function

$$
\alpha_{i}=-z_{i}-2(n-i+1) z_{i}-\Omega_{i} z_{i}-\Lambda_{i} z_{i} \triangleq-\beta_{i} z_{i},
$$

then (39) can be changed into

$$
\begin{aligned}
\dot{V}_{i} \leq & \frac{1}{2} z_{i+1}^{2}+\pi_{i}\left|\xi_{i}\right|^{4}+\sum_{j=1}^{i-1}\left(\pi_{j}+\sum_{l=j+1}^{i} \pi_{l j}\right)\left|\xi_{j}\right|^{4}-\sum_{j=1}^{i} V_{\tau j} \\
& -(n-i) e^{-\tau}\left(1-\tau^{*}\right) \sum_{j=1}^{i} z_{j}^{2}(t-\tau(t)) \\
& -(n-i+1) \sum_{j=1}^{i} z_{j}^{2} .
\end{aligned}
$$

Let $i=n$, together with $z_{n+1}=0$, then one gets the actual control law

$$
u=-z_{n}-2 z_{n}-\Omega_{n} z_{n}-\Lambda_{n} z_{n}=-\sum_{i=1}^{n}\left(\prod_{j=i}^{n} \beta_{j}\right) x_{i},
$$

and

$$
\begin{aligned}
\dot{V}_{n} \leq & -\sum_{j=1}^{n} \frac{1}{2} z_{j}^{2}-\sum_{j=1}^{n} V_{\tau j}+\pi_{n}\left|\xi_{n}\right|^{4} \\
& +\sum_{j=1}^{n-1}\left(\pi_{j}+\sum_{l=j+1}^{n} \pi_{l j}\right)\left|\xi_{j}\right|^{4} \\
\leq & -V_{n}+\tilde{\pi}|\xi|^{4}
\end{aligned}
$$

where $\tilde{\pi}=\max \left\{\max _{1 \leq j \leq n-1}\left\{\pi_{j}+\sum_{l=j+1}^{n} \pi_{l j}\right\}, \pi_{n}\right\}$. 
According to the above design procedure, for system 27. satisfying the assumptions $\mathbf{H 0}, \mathbf{H 1}$ and $\mathbf{H 2}$, one chooses the Lyapunov-Krasovskii functional

$V(t, z(t))=\sum_{i=1}^{n}\left[\frac{1}{2} z_{i}^{2}(t)+(n-i+1) \int_{t-\tau(t)}^{t} e^{s-t} z_{i}^{2}(s) d s\right]$.

It is clear that

$$
V(t, z(t)) \geq \sum_{j=1}^{n} \frac{1}{2} z_{j}^{2}=\frac{1}{2}|z(t)|^{2}
$$

and

$$
\begin{aligned}
V(t, z(t)) & \leq n \sum_{j=1}^{n} \int_{-\tau(t)}^{0} z_{j}^{2}(v+t) d v+\sum_{j=1}^{n} \frac{1}{2} z_{j}^{2} \\
& \leq n^{2} \tau \sum_{j=1}^{n}\left(\sup _{-\tau \leq v \leq 0}\left|z_{j}(v+t)\right|\right)^{2}+\frac{1}{2}|z(t)|^{2} \\
& \leq \frac{1+2 n^{2} \tau}{2}\left(\sup _{-\tau \leq v \leq 0}|z(v+t)|\right)^{2},
\end{aligned}
$$

that is to say,

$$
\frac{1}{2}|z(t)|^{2} \leq V(t, z(t)) \leq \frac{1+2 n^{2} \tau}{2}\left(\sup _{-\tau \leq v \leq 0}|z(v+t)|\right)^{2} .
$$

From (41) and 421, the Lyapunov-Krasovskii functional $V$ meets the requirements of Theorem 1 so the closed-loop system (including (27) and (40) ) is noise-to-state stable in the mean-square sense and has a unique solution which is UB-2-M. Moreover, the output regulation error of closed-loop system satisfies

$$
\lim _{t \rightarrow \infty} E|y(t)|^{2} \leq \lim _{t \rightarrow \infty} E|z(t)|^{2} \leq 2 \tilde{\pi} K .
$$

According to the definitions of $\tilde{\pi}$, the regulation error can be decreased by tuning the positive constants $\pi_{j}+$ $\sum_{i=j+1}^{n} \pi_{i j}(1 \leq j \leq n-1)$ and $\pi_{n}$ small enough (i.e., letting $\pi_{j}, \pi_{i j}(i=j+1,1 \leq j \leq n-1)$ and $\pi_{n}$ arbitrarily small $)$, where $\pi_{j}(1 \leq j \leq n-1), \pi_{i j}(i=j+1,1 \leq j \leq n-1)$ and $\pi_{n}$ are independent of each other. In other words, the regulation error can be made small enough in the mean-square sense by selecting appropriate design parameters.

From what has been analyzed above, one draws the stability analysis result as below at present.

Theorem 3: If system 27] satisfies the assumptions Ho, $\mathbf{H 1}$ and $\mathbf{H 2}$, then by selecting appropriate design parameters, the closed-loop system (27) and (40) is NSS-2-M and has a unique solution which is UB-2-M. Moreover, the regulation error satisfies

$$
\lim _{t \rightarrow \infty} E|y(t)|^{2} \leq 2 \tilde{\pi} K,
$$

and the regulation error can be made arbitrarily small in the mean-square sense by parameter-tuning technique.

\section{APPLICATION 2: ADAPTIVE OUTPUT FEEDBACK REGULATION CONTROL}

In this section, one focuses on the adaptive output feedback regulation control for system (27). To this end, one supposes that system 27] satisfies the assumption $\mathbf{H O}$ and the following two assumptions:

H1': Only $x_{1}(t)$ is measurable by system output $y(t)$, other state variables $x_{2}(t), \cdots, x_{n}(t)$ are unavailable.

H2': For $i=1,2, \cdots, n$, unknown functions $f_{i}, g_{i}$ satisfy

$\left|f_{i}\left(t, \bar{x}_{i}(t-\tau(t)), \bar{x}_{i}(t)\right)\right| \leq \theta_{i}\left[\phi_{\tau i}(y(t-\tau(t)))+\phi_{i}(y(t))\right]$, $\left|g_{i}\left(t, \bar{x}_{i}(t-\tau(t)), \bar{x}_{i}(t)\right)\right| \leq \theta_{i}\left[\psi_{\tau i}(y(t-\tau(t)))+\psi_{i}(y(t))\right]$,

where $\phi_{i}, \phi_{\tau i}, \psi_{i}$ and $\psi_{\tau i}$ are smooth non-negative function with $\phi_{i}(0)=\phi_{\tau i}(0)=\psi_{i}(0)=\psi_{\tau i}(0)=0 . \theta_{i}>0$ are unknown parameters.

It follows from the assumption $\mathbf{H 2}$ ' that there exist smooth functions $\bar{\phi}_{i}(y), \bar{\phi}_{\tau i}(y(t-\tau(t))), \bar{\psi}_{i}(y)$ and $\bar{\psi}_{\tau i}(y(t-\tau(t)))$ satisfying

$$
\begin{aligned}
& \phi_{i}(y)=y \bar{\phi}_{i}(y), \quad \psi_{i}(y)=y \bar{\psi}_{i}(y), \\
& \phi_{\tau i}(y(t-\tau(t)))=y(t-\tau(t)) \bar{\phi}_{\tau i}(y(t-\tau(t))), \\
& \psi_{\tau i}(y(t-\tau(t)))=y(t-\tau(t)) \bar{\psi}_{\tau i}(y(t-\tau(t))) .
\end{aligned}
$$

The task in this section is to construct a regulation controller for system (27) by adaptive output feedback method such that the system output $y(t)$ can be regulated to an neighborhood of the zero whose scope can be controlled and the system state is bounded in the mean-square sense.

\section{A. Observer design}

Since state variables $x_{2}(t), \cdots, x_{n}(t)$ are unavailable, one reconfigures $x_{i}(t)(i=2, \cdots, n)$ by the following reducedorder observer:

$$
\left\{\begin{array}{l}
\dot{\hat{x}}_{i}(t)=\hat{x}_{i+1}(t)+\kappa_{i+1} y(t)-\kappa_{i}\left(\hat{x}_{1}(t)+\kappa_{1} y(t)\right), \\
i=1,2, \ldots n-2, \\
\dot{\hat{x}}_{n-1}(t)=u(t)-\kappa_{n-1}\left(\hat{x}_{1}(t)+\kappa_{1} y(t)\right),
\end{array}\right.
$$

where $\kappa_{1}, \ldots, \kappa_{n-1}$ are the observer gain to be designed.

Let $\theta^{*}=\max \left\{1, \theta_{1}, \cdots, \theta_{n}\right\}, e=\left[e_{2}, \cdots, e_{n}\right]^{T}$ with $e_{j}=$ $\left(x_{j}(t)-\hat{x}_{j-1}(t)-\kappa_{j-1} y(t)\right) / \theta^{*}(j=2,3, \cdots, n)$, then the dynamic trajectory of observer error can be described by

$$
\dot{e}=A_{e} e+\frac{1}{\theta^{*}} \Xi_{1}+\frac{1}{\theta^{*}} \Xi_{2} \xi,
$$

where $A_{e}=\left(-\kappa, \bar{I}_{n-1}\right), \bar{I}_{n-1}=\operatorname{diag}\{1,1, \cdots, 1,0\}, \kappa=$ $\left(\kappa_{1}, \cdots, \kappa_{n-1}\right)^{T}, \Xi_{1}=\left[f_{2}-\kappa_{1} f_{1}, \cdots, f_{n}-\kappa_{n-1} f_{1}\right]^{T}, \Xi_{2}=$ $\left(-g_{1} \kappa, \Theta\right), \Theta=\operatorname{diag}\left\{g_{2}, \cdots, g_{n}\right\}$.

The observer gain $\kappa_{1}, \ldots, \kappa_{n-1}$ should be chosen to meet the requirement that $A_{e}$ is Hurwitz. This means that there is a matrix $P_{n-1}>0$ such that $A_{e}^{T} P_{n-1}+P_{n-1} A_{e}+b I_{n-1}=0$, where $b$ is a positive design constant.

\section{B. Adaptive regulation controller design}

First, one introduces the error transformations

$$
z_{1}=y(t), \quad z_{i+1}=\hat{x}_{i}-\alpha_{i}\left(y, \overline{\hat{x}}_{i-1}, \hat{\theta}\right),
$$

where $\overline{\hat{x}}_{i-1}(t)=\left(\hat{x}_{1}(t), \cdots, \hat{x}_{i-1}(t)\right)^{T}, i=1,2, \cdots, n$, $\hat{x}_{n}=u, z_{n+1}=0, \overline{\hat{x}}_{0}=0, \alpha_{i}(i=1, \cdots, n)$ are stabilizing functions to be determined and $\hat{\theta}$ is the estimation of $\theta$ with $\theta=\max \left\{\theta^{* 2}, \theta^{* 4}\right\}$. In the light of (44)-(46), one can deduce that

$$
\dot{z}_{1}=e_{2} \theta^{*}+\hat{x}_{1}+\kappa_{1} y+f_{1}+g_{1} \xi_{1},
$$




$$
\begin{gathered}
\dot{z}_{i}=z_{i+1}+\alpha_{i}-\frac{\partial \alpha_{i-1}}{\partial \hat{\theta}} \dot{\hat{\theta}}-\frac{\partial \alpha_{i-1}}{\partial y}\left(e_{2} \theta^{*}+f_{1}\right) \\
-\frac{\partial \alpha_{i-1}}{\partial y} g_{1} \xi_{1}+\bar{\beta}_{i}, \quad i=2, \cdots, n,
\end{gathered}
$$

where $\bar{\beta}_{i}=\kappa_{i} y-\sum_{j=1}^{i-2} \frac{\partial \alpha_{i-1}}{\partial \hat{x}_{j}}\left(\hat{x}_{j+1}+\kappa_{j+1} y-\kappa_{i}\left(\hat{x}_{1}+\kappa_{1} y\right)\right)-$ $\left(\kappa_{i-1}+\frac{\partial \alpha_{i-1}}{\partial y}\right)\left(\hat{x}_{1}+\kappa_{1} y\right)$.

Next, one begins to design adaptive regulation controller.

Step 1 Selecting the candidate Lyapunov-Krasovskii functional

$$
\begin{aligned}
& V_{1}=V_{0}+V_{\tau}+\frac{1}{2} z_{1}^{2}, \quad V_{0}=e^{T} P e+\frac{1}{2 \mu} \tilde{\theta}^{2}, \\
& V_{\tau}=\int_{t-\tau(t)}^{t} e^{s-t} Q(y(s)) d s,
\end{aligned}
$$

where $\tilde{\theta}=\theta-\hat{\theta}$, both the adaptive gain $\mu>0$ and continuous function $Q(y(s))>0$ are to be designed. By (47), $\dot{\tau}(t) \leq$ $\tau^{*}<1$ and $0 \leq \tau(t) \leq \tau$, the derivative of $V_{1}$ satisfies that

$$
\begin{aligned}
\dot{V}_{1}= & \dot{V}_{0}+z_{1}\left(e_{2} \theta^{*}+\hat{x}_{1}+\kappa_{1} y+f_{1}+g_{1} \xi_{1}\right)-V_{\tau} \\
& +Q(y(t))-e^{-\tau(t)}(1-\dot{\tau}(t)) Q(y(t-\tau(t))) \\
\leq & -b|e|^{2}+\frac{2}{\theta^{*}} e^{T} P \Xi_{1}+\frac{2}{\theta^{*}} e^{T} P \Xi_{2} \xi-\frac{1}{\mu} \tilde{\theta} \dot{\hat{\theta}}-V_{\tau} \\
& -e^{-\tau}\left(1-\tau^{*}\right) Q(y(t-\tau(t)))+Q(y)+z_{1} e_{2} \theta^{*} \\
& +z_{1}\left(z_{2}+\alpha_{1}+\kappa_{1} y\right)+z_{1} f_{1}+z_{1} g_{1} \xi_{1} .
\end{aligned}
$$

It follows from assumption H2', 437, Lemma 7 and Young's inequality that

$$
\begin{aligned}
z_{1} e_{2} \theta^{*} \leq & d_{11}|e|^{2}+\frac{1}{4} d_{11}^{-1} z_{1}^{2} \theta \\
z_{1} f_{1} \leq & \frac{1}{4} d_{12}^{-1} z_{1}^{2} \theta+2 d_{12} y^{2} \bar{\phi}_{1}^{2}+2 d_{12} \phi_{\tau 1}^{2} \\
z_{1} g_{1} \xi_{1} \leq & \frac{1}{8} d_{13}^{-1} z_{1}^{4} \theta+d_{13}^{-1} y^{4} \bar{\psi}_{1}^{4} \\
& +d_{13}^{-1} \psi_{\tau 1}^{4}+d_{13}|\xi|^{2} \\
\frac{2}{\theta^{*}} e^{T} P \Xi_{1} \leq & \sum_{i=2}^{n}\left[\left(\bar{\phi}_{i}^{2}+\kappa_{i-1}^{2} \bar{\phi}_{1}^{2}\right) y^{2}+\left(\phi_{\tau i}^{2}+\kappa_{i-1}^{2} \phi_{\tau 1}^{2}\right)\right] \\
& \left.\cdot 4\|P\|\right|_{F} ^{2} d_{01}^{-1}+d_{01}|e|^{2} \\
\frac{2}{\theta^{*}} e^{T} P \Xi_{2} \xi & \leq \sum_{i=2}^{n}\left[\left(\bar{\psi}_{i}^{4}+\kappa_{i-1}^{4} \bar{\psi}_{1}^{4}\right) y^{4}+\left(\psi_{\tau i}^{4}+\kappa_{i-1}^{4} \psi_{\tau 1}^{4}\right)\right] \\
& \left.\cdot 4(n-1) d_{02}^{-1}|| P\left|\|_{F}^{4}+\right| e\right|^{2}+d_{02}|\xi|^{4}
\end{aligned}
$$

where $d_{01}, d_{02}, d_{11}, d_{12}$ and $d_{13}$ are positive design parameters. Substituting (50)-(54) into 49), one obtains

$$
\begin{aligned}
\dot{V}_{1} \leq & -b_{1}|e|^{2}+z_{1}\left(z_{2}+\alpha_{1}+\kappa_{1} y+\omega+\frac{1}{8} \varpi_{1} \hat{\theta}\right)+\delta_{1} \\
& +\left(\frac{1}{8} \varpi_{1} z_{1}-\frac{1}{\mu} \dot{\hat{\theta}}\right) \tilde{\theta}+d_{02}|\xi|^{4}+d_{13}|\xi|^{2}-V_{\tau} \\
& -e^{-\tau}\left(1-\tau^{*}\right) Q(y(t-\tau(t)))+Q(y),
\end{aligned}
$$

where $\omega=4\|P\|_{F}^{2} d_{01}^{-1} \sum_{i=2}^{n}\left[\bar{\phi}_{i}^{2}+\kappa_{i-1}^{2} \bar{\phi}_{1}^{2}\right] y+4(n-$ 1) $\|P\|_{F}^{4} d_{02}^{-1} \sum_{i=2}^{n}\left[\bar{\psi}_{i}^{4}+\kappa_{i-1}^{4} \bar{\psi}_{1}^{4}\right] y^{3}+2 d_{12} \bar{\phi}_{1}^{2} y+d_{13}^{-1} \bar{\psi}_{1}^{4} y^{3}$, $\delta_{1}=\delta_{0}+2 d_{12} \phi_{\tau 1}^{2}+d_{13}^{-1} \psi_{\tau 1}^{4}, \delta_{0}=4\|P\|_{F}^{2} d_{01}^{-1} \sum_{i=2}^{n}\left[\phi_{\tau i}^{2}+\right.$ $\left.\kappa_{i-1}^{2} \phi_{\tau 1}^{2}\right]+4(n-1)\|P\|_{F}^{4} d_{02}^{-1} \sum_{i=2}^{n}\left[\psi_{\tau i}^{4}+\kappa_{i-1}^{4} \psi_{\tau 1}^{4}\right], \varpi_{1}=$ $2 d_{11}^{-1} z_{1}+2 d_{12}^{-1} z_{1}+d_{13}^{-1} z_{1}^{3}, b_{1}=b_{0}-d_{11}>0, b_{0}=$ $b-d_{01}-1>0$.
Selecting the following tuning function $\lambda_{1}$ and the stabilizing function $\alpha_{1}$ respectively

$$
\begin{aligned}
\lambda_{1} & =\frac{1}{8} \varpi_{1} z_{1}-\frac{1}{\mu} \hat{\theta}, \\
\alpha_{1}(y, \hat{\theta}) & =-c_{1} z_{1}-\kappa_{1} y-\omega-\frac{1}{8} \varpi_{1} \hat{\theta}-\sigma(y),
\end{aligned}
$$

where positive constant $c_{1}$ and the function $\sigma(y)$ are to be designed, then

$$
\begin{aligned}
\dot{V}_{1} \leq & -b_{1}|e|^{2}+z_{1} z_{2}-c_{1} z_{1}^{2}-\sigma(y) y+\left(\lambda_{1}-\frac{1}{\mu} \dot{\hat{\theta}}\right) \tilde{\theta} \\
& +\frac{1}{\mu} \hat{\theta} \tilde{\theta}+\delta_{1}+d_{02}|\xi|^{4}+d_{13}|\xi|^{2}-V_{\tau} \\
& -e^{-\tau}\left(1-\tau^{*}\right) Q(y(t-\tau(t)))+Q(y) .
\end{aligned}
$$

Step $\mathbf{k}(k=2, \cdots, n)$ Suppose that the appropriate stabilizing functions $\alpha_{j}(j=1,2, \cdots, k-1)$ and tuning functions $\lambda_{j}(j=1,2, \cdots, k-1)$ have been designed such that $V_{k-1}=V_{k-2}+\frac{1}{2} z_{k-1}^{2}$ satisfies

$$
\begin{aligned}
\dot{V}_{k-1} \leq & -b_{k-1}|e|^{2}-\sum_{j=1}^{k-1} c_{j} z_{j}^{2}+z_{k-1} z_{k}-\sigma(y) y \\
& +d_{02}|\xi|^{4}+\sum_{j=1}^{k-1} d_{j 3}|\xi|^{2}+\frac{1}{\mu} \hat{\theta} \tilde{\theta}+\delta_{k-1} \\
& +\sum_{j=2}^{k-1}\left[2 d_{j 2} \bar{\phi}_{1}^{2}+d_{13}^{-1} \bar{\psi}_{1}^{4} y^{2}\right] y^{2}+\left(\lambda_{k-1}-\frac{1}{\mu} \dot{\hat{\theta}}\right) \tilde{\theta} \\
& +\mu \sum_{j=2}^{k-1} z_{j} \frac{\partial \alpha_{j-1}}{\partial \hat{\theta}}\left(\lambda_{k-1}-\frac{1}{\mu} \dot{\hat{\theta}}\right)-V_{\tau} \\
& -e^{-\tau}\left(1-\tau^{*}\right) Q(y(t-\tau(t)))+Q(y),
\end{aligned}
$$

where $\delta_{k-1}=\delta_{k-2}+2 d_{(k-1) 2} \phi_{\tau 1}^{2}+d_{(k-1) 3}^{-1} \psi_{\tau 1}^{4}, b_{k-1}=$ $b_{k-2}-d_{(k-1) 1}>0$.

In what follows, one selects $V_{k}=V_{k-1}+\frac{1}{2} z_{k}^{2}$ in Step k to prove that $V_{k}$ also satisfies (57). From (48) and (57), one has

$$
\begin{aligned}
\dot{V}_{k} \leq & -b_{k-1}|e|^{2}-\sum_{j=1}^{k-1} c_{j} z_{j}^{2}+\delta_{k-1}+d_{02}|\xi|^{4} \\
& +\sum_{j=1}^{k-1} d_{j 3}|\xi|^{2}+z_{k}\left(z_{k-1}+z_{k+1}+\alpha_{k}+\bar{\beta}_{k}\right) \\
& -z_{k} \frac{\partial \alpha_{k-1}}{\partial y}\left(e_{2} \theta^{*}+f_{1}+g_{1} \xi_{1}\right)-z_{k} \frac{\partial \alpha_{k-1} \dot{\hat{\theta}}}{\partial \hat{\theta}} \\
& +\sum_{j=2}^{k-1}\left[2 d_{j 2} \bar{\phi}_{1}^{2}+d_{j 3}^{-1} \bar{\psi}_{1}^{4} y^{2}\right] y^{2}+\frac{1}{\mu} \hat{\theta} \tilde{\theta}-\sigma(y) y \\
& +\left(\lambda_{k-1}-\frac{1}{\mu} \dot{\hat{\theta}}\right) \tilde{\theta}+\mu \sum_{j=2}^{k-1} z_{j} \frac{\partial \alpha_{j-1}}{\partial \hat{\theta}}\left(\lambda_{k-1}-\frac{1}{\mu} \dot{\hat{\theta}}\right) \\
& -V_{\tau}-e^{-\tau}\left(1-\tau^{*}\right) Q(y(t-\tau(t)))+Q(y) .
\end{aligned}
$$

By Young's inequality, assumption H2' and 433, one gets the following inequalities

$$
-z_{k} \frac{\partial \alpha_{k-1}}{\partial y} e_{2} \theta^{*} \leq d_{k 1}|e|^{2}+\frac{1}{4} d_{k 1}^{-1} z_{k}^{2}\left(\frac{\partial \alpha_{k-1}}{\partial y}\right)^{2} \theta,
$$




$$
\begin{aligned}
-z_{k} \frac{\partial \alpha_{k-1}}{\partial y} f_{1} \leq & \frac{1}{4} d_{k 2}^{-1} z_{k}^{2}\left(\frac{\partial \alpha_{k-1}}{\partial y}\right)^{2} \theta+2 d_{k 2} y^{2} \bar{\phi}_{1}^{2} \\
& +2 d_{k 2} \phi_{\tau 1}^{2} \\
-z_{k} \frac{\partial \alpha_{k-1}}{\partial y} g_{1} \xi_{1} \leq & \frac{1}{8} d_{k 3}^{-1} z_{k}^{4}\left(\frac{\partial \alpha_{k-1}}{\partial y}\right)^{4} \theta+d_{k 3}^{-1} y^{4} \bar{\psi}_{1}^{4} \\
& +d_{k 3}^{-1} \psi_{\tau 1}^{4}+d_{k 3}|\xi|^{2} .
\end{aligned}
$$

Substituting 59)- 610 into 587, then $\dot{V}_{k}$ satisfies

$$
\begin{aligned}
\dot{V}_{k} \leq & -b_{k}|e|^{2}-\sum_{j=1}^{k-1} c_{j} z_{j}^{2}+\left(\frac{1}{8} z_{k} \varpi_{k}+\lambda_{k-1}-\frac{1}{\mu} \dot{\hat{\theta}} \tilde{\theta}\right. \\
& +z_{k}\left(z_{k-1}+z_{k+1}+\alpha_{k}+\bar{\beta}_{k}+\frac{1}{8} \varpi_{k} \hat{\theta}-\mu \frac{\partial \alpha_{k-1}}{\partial \hat{\theta}} \lambda_{k}\right) \\
& -\sigma(y) y+\delta_{k}+\mu z_{k} \frac{\partial \alpha_{k-1}}{\partial \hat{\theta}}\left(\lambda_{k}-\frac{1}{\mu} \dot{\hat{\theta}}\right)+\frac{1}{\mu} \hat{\theta} \tilde{\theta} \\
& +\mu \sum_{j=2}^{k-1} z_{j} \frac{\partial \alpha_{j-1}}{\partial \hat{\theta}}\left(\lambda_{k-1}-\frac{1}{\mu} \dot{\hat{\theta}}\right)+d_{02}|\xi|^{4} \\
& +\sum_{j=1}^{k} d_{j 3}|\xi|^{2}+\sum_{j=2}^{k}\left[2 d_{j 2} \bar{\phi}_{1}^{2}+d_{j 3}^{-1} \bar{\psi}_{1}^{4} y^{2}\right] y^{2} \\
& -e^{-\tau}\left(1-\tau^{*}\right) Q(y(t-\tau(t)))+Q(y)-V_{\tau},
\end{aligned}
$$

where $d_{k 1}, d_{k 2}$ and $d_{k 3}$ are positive design constants. $b_{k}=$ $b_{k-1}-d_{k 1}>0, \varpi_{k}=2 d_{k 1}^{-1} z_{k}\left(\frac{\partial \alpha_{k-1}}{\partial y}\right)^{2}+2 d_{k 2}^{-1} z_{k}\left(\frac{\partial \alpha_{k-1}}{\partial y}\right)^{2}+$ $d_{k 3}^{-1} z_{k}^{3}\left(\frac{\partial \alpha_{k-1}}{\partial y}\right)^{4}$ and $\delta_{k}=\delta_{k-1}+2 d_{k 2} \phi_{\tau 1}^{2}+d_{k 3}^{-1} \psi_{\tau 1}^{4}$.

First, let the tuning function $\lambda_{k}=\lambda_{k-1}+\frac{1}{8} z_{k} \varpi_{k}$, then

$$
\begin{aligned}
& \frac{1}{8} z_{k} \varpi_{k} \tilde{\theta}+\left(\lambda_{k-1}-\frac{1}{\mu} \dot{\hat{\theta}}\right) \tilde{\theta}=\left(\lambda_{k}-\frac{1}{\mu} \dot{\hat{\theta}}\right) \tilde{\theta} \\
& \mu \sum_{j=2}^{k-1} z_{j} \frac{\partial \alpha_{j-1}}{\partial \hat{\theta}}\left(\lambda_{k}-\frac{1}{\mu} \dot{\hat{\theta}}\right)-\frac{1}{8} z_{k} \varpi_{k} \mu \sum_{j=2}^{k-1} z_{j} \frac{\partial \alpha_{j-1}}{\partial \hat{\theta}} \\
= & \mu \sum_{j=2}^{k-1} z_{j} \frac{\partial \alpha_{j-1}}{\partial \hat{\theta}}\left(\lambda_{k-1}-\frac{1}{\mu} \dot{\hat{\theta}}\right) .
\end{aligned}
$$

Next, one substitutes 637-64 into 62 and chooses the stabilizing function

$$
\begin{aligned}
\alpha_{k}\left(y, \overline{\hat{x}}_{k-1}, \hat{\theta}\right)= & -c_{k} z_{k}-z_{k-1}-\bar{\beta}_{k}+\mu \frac{\partial \alpha_{k-1}}{\partial \hat{\theta}} \lambda_{k} \\
& -\frac{1}{8} \varpi_{k} \hat{\theta}+\frac{1}{8} \varpi_{k} \mu \sum_{j=2}^{k-1} z_{j} \frac{\partial \alpha_{j-1}}{\partial \hat{\theta}},
\end{aligned}
$$

then (62) can be changed into

$$
\begin{aligned}
\dot{V}_{k} \leq & -b_{k}|e|^{2}-\sum_{j=1}^{k} c_{j} z_{j}^{2}+z_{k} z_{k+1}+\left(\lambda_{k}-\frac{1}{\mu} \dot{\hat{\theta}}\right) \tilde{\theta} \\
& +\mu \sum_{j=2}^{k} z_{j} \frac{\partial \alpha_{j-1}}{\partial \hat{\theta}}\left(\lambda_{k}-\frac{1}{\mu} \dot{\hat{\theta}}\right)+\frac{1}{\mu} \hat{\theta} \tilde{\theta}+d_{02}|\xi|^{4}+\delta_{k} \\
& +\sum_{j=1}^{k} d_{j 3}|\xi|^{2}-\sigma(y) y+\sum_{j=2}^{k}\left[2 d_{j 2} \bar{\phi}_{1}^{2}+d_{j 3}^{-1} \bar{\psi}_{1}^{4} y^{2}\right] y^{2} \\
& -e^{-\tau}\left(1-\tau^{*}\right) Q(y(t-\tau(t)))+Q(y)-V_{\tau} .
\end{aligned}
$$

At Step n, let $Q(y)=\bar{Q}(y) y, \sigma(y)=\bar{Q}(y)+$ $\sum_{j=2}^{n}\left[2 d_{j 2} \bar{\phi}_{1}^{2}(y)+d_{j 3}^{-1} \bar{\psi}_{1}^{4}(y) y^{2}\right] y$, where

$$
\begin{aligned}
\bar{Q}(y(t))= & \frac{4 e^{\tau}}{1-\tau^{*}}\|P\|_{F}^{2} d_{01}^{-1} y \sum_{i=2}^{n}\left[\bar{\phi}_{\tau i}^{2}(y)+\kappa_{i-1}^{2} \bar{\phi}_{\tau 1}^{2}(y)\right] \\
& +\frac{e^{\tau}}{1-\tau^{*}} \sum_{j=1}^{n}\left[2 d_{j 2} \bar{\phi}_{\tau 1}^{2}(y)+d_{j 3}^{-1} \bar{\psi}_{\tau 1}^{4}(y) y^{2}\right] y \\
& +\frac{4(n-1) e^{\tau}}{1-\tau^{*}}\|P\|_{F}^{4} d_{02}^{-1} y^{3} \\
& \cdot \sum_{i=2}^{n}\left[\bar{\psi}_{\tau i}^{4}(y)+\kappa_{i-1}^{4} \bar{\psi}_{\tau 1}^{4}(y)\right] .
\end{aligned}
$$

Furthermore, selecting the update law

$$
\dot{\hat{\theta}}=\mu \lambda_{n}, \quad \lambda_{n}=\lambda_{n-1}+\frac{1}{8} z_{n} \varpi_{n},
$$

and the control law

$$
\begin{aligned}
u= & -c_{n} z_{n}-z_{n-1}-\bar{\beta}_{n}-\frac{1}{8} \varpi_{n} \hat{\theta}+\mu \frac{\partial \alpha_{n-1}}{\partial \hat{\theta}} \lambda_{n} \\
& +\frac{1}{8} \varpi_{n} \mu \sum_{j=2}^{n-1} z_{j} \frac{\partial \alpha_{j-1}}{\partial \hat{\theta}},
\end{aligned}
$$

then one has

$$
\begin{aligned}
\dot{V}_{n} \leq & -b_{n}|e|^{2}-\sum_{j=1}^{n} c_{j} z_{j}^{2}+\frac{1}{\mu} \hat{\theta} \tilde{\theta}+d_{02}|\xi|^{4} \\
& +\sum_{j=1}^{n} d_{j 3}|\xi|^{2}-V_{\tau} \\
\leq & -\frac{b_{n}}{\sigma_{M}} e^{T} P e-\frac{1}{2 \mu} \tilde{\theta}^{2}-V_{\tau}-\sum_{j=1}^{n} c_{j} z_{j}^{2}+\frac{1}{2 \mu} \theta^{2} \\
& +d_{02}|\xi|^{4}+\sum_{j=1}^{n} d_{j 3}\left(\frac{1}{4}+|\xi|^{4}\right) \\
\leq & -\bar{c} V_{n}+\bar{d}_{1}|\xi|^{4}+\bar{d}_{2},
\end{aligned}
$$

where $\sigma_{M}=\lambda_{\max }(P), \bar{c}=\min \left\{\frac{b_{n}}{\sigma_{M}}, 1,2 c_{1}, 2 c_{2}, \cdots, 2 c_{n}\right\}$, $\bar{d}_{1}=d_{02}+\sum_{j=1}^{n} d_{j 3}$, and $\bar{d}_{2}=\frac{1}{2 \mu} \theta^{2}+\frac{1}{4} \sum_{j=1}^{n} d_{j 3}$.

\section{Stability analysis}

Theorem 4: Suppose that assumptions H0, H1' and H2' hold for system (27), then the closed-loop system including 27), 66 and 678 is NSS-2-M and the closed-loop system solution on $\left[t_{0}-\tau, \infty\right)$ is UB-2-M. Furthermore, the regulation error in the mean-square sense satisfies

$$
\lim _{t \rightarrow \infty} E|y(t)|^{2} \leq \frac{2 \bar{d}_{1} K+2 \bar{d}_{2}}{\bar{c}},
$$

and by selecting appropriate design parameters, the upper bound of the regulation error can be made arbitrarily small.

Proof: For system [27), one chooses the LyapunovKrasovskii functional

$$
V=e^{T} P e+\frac{1}{2 \mu} \tilde{\theta}^{2}+\sum_{j=1}^{n} \frac{1}{2} z_{j}^{2}+\int_{t-\tau(t)}^{t} e^{s-t} Q(y(s)) d s,
$$


from which one knows that $V$ is a radially unbounded functional and

$$
\dot{V} \leq-\bar{c} V+\bar{d}_{1}|\xi|^{4}+\bar{d}_{2}
$$

where $\bar{c}=\min \left\{\frac{b_{n}}{\sigma_{M}}, 1,2 c_{1}, 2 c_{2}, \cdots, 2 c_{n}\right\}, \bar{d}_{1}=d_{02}+$ $\sum_{j=1}^{n} d_{j 3}$, and $\bar{d}_{2}=\frac{1}{2 \mu} \theta^{2}+\frac{1}{4} \sum_{j=1}^{n} d_{j 3}$ are all positive constants. Taking expectations on both sides of 69), one obtains

$$
E \dot{V} \leq-\bar{c} E V+\bar{d}_{1} E|\xi(t)|^{4}+\bar{d}_{2} .
$$

By Lemma 4 the closed-loop system including 27, 66 and (67) has a unique solution.

Defining $\tilde{\nu}(t)=E V$ and $z(t)=\left[z_{1}(t), \cdots, z_{n}(t)\right]^{T}$, one adopts the same way for 69 as one has done for (19) in the proof of Theorem 1] then the inequality $\tilde{\nu}(t) \leq$ $\tilde{\nu}\left(t_{0}\right) e^{-\bar{c}\left(t-t_{0}\right)}+\left(\bar{d}_{1} K+\bar{d}_{2}\right) / \bar{c}$ holds, together with $V \geq$ $\frac{1}{2}|z(t)|^{2}$, one gets

$$
E|z(t)|^{2} \leq 2 E\left(\left.V\right|_{t=t_{0}}\right) e^{-\bar{c}\left(t-t_{0}\right)}+\frac{2 \bar{d}_{1} K+2 \bar{d}_{2}}{\bar{c}}
$$

which implies that the closed-loop system including 27, 66 and 67 is NSS-2-M. Furthermore, letting $t \rightarrow \infty$ in 70. yields

$$
\lim _{t \rightarrow \infty} E|z(t)|^{2} \leq \frac{2 \bar{d}_{1} K+2 \bar{d}_{2}}{\bar{c}} .
$$

This means that the state of the closed-loop system including (27), 66) and 67) is UB-2-M.

Additionally, it follows from (71) that

$$
\lim _{t \rightarrow \infty} E|y(t)|^{2} \leq \lim _{t \rightarrow \infty} E|z(t)|^{2} \leq \frac{2 \bar{d}_{1} K+2 \bar{d}_{2}}{\bar{c}},
$$

which implies that the upper bound of regulation error in the mean-square sense can be made small enough by selecting $d_{02}, d_{j 3}(1 \leq j \leq n)$ sufficiently small and $\mu$ large enough. The parameters consisting of $\bar{c}, d_{02}, \mu, d_{j 3}(1 \leq j \leq n)$ are independent from each other. One completes the proof.

Remark 4: From the above design procedure of adaptive output feedback regulation controller in this section, the observer is introduced to estimate the unmeasurable states, which lead to the assumption $\mathbf{H 0}$ is proposed. If the state of system 27) is measurable (i.e., the assumption $\mathbf{H 1}$ holds for system (27)), and system 27) satisfies the assumptions H0' (the random disturbance $\xi(t)$ satisfies $\sup _{t \geq t_{0}} E|\xi(t)|^{2}<K$ ) and H2', then one can design an adaptive state feedback controller to achieve regulation control objective. The design procedure of state feedback controller is as similar as the procedure given above, thus one omits the detailed process.

\section{Simulation EXAmples}

Example 1: The time-delay phenomenon widespreadly exists in the chemical industry, such as the chemical reactor recycle system ([23], [24]). Consider a two-stage chemical reactor system that is modeled as

$$
\left\{\begin{array}{l}
\dot{x}_{1}=-l_{1}(t) x_{1}-\frac{1}{\mu_{1}} x_{1}+\frac{1-r_{1}}{v_{1}} x_{2}, \\
\dot{x}_{2}=\frac{\lambda_{2}}{v_{2}} u-l_{2}(t) x_{2}-\frac{1}{\mu_{2}} x_{2}+\frac{r_{1}}{v_{2}} x_{1 t}+\frac{r_{2}}{v_{2}} x_{2 t}, \\
y=x_{1}
\end{array}\right.
$$

where $x_{1 t}=x_{1}(t-\tau(t))$ and $x_{2 t}=x_{2}(t-\tau(t)), x_{1}$ and $x_{2}$ stand for produced streams from the two reactors; the residence time of two reactors are $\mu_{1}$ and $\mu_{2} ; v_{1}$ and $v_{2}$ represent reactor volumes; $r_{1}$ and $r_{2}$ denote the recycle flow rates; the feed rate is $\lambda_{2} ; \tau(t)=0.1(1-\sin t)$ represents the time delay; $l_{1}(t)$ and $l_{2}(t)$ indicate the uncertain reaction functions.

For system 272, [24 studies the impact of the white noise random disturbance for the reactors by introducing It $\hat{o}$ stochastic differential equation. Here, one considers random differential equation by introducing random noise disturbance that is not white noise. Let the uncertain reaction functions $l_{i}(t)=l_{i 0}+\xi_{i}(t) / \sqrt{\left|x_{i}(t)\right|}, i=1,2$, where $l_{i 0}$ are reaction constants and random disturbance $\xi(t)=\left[\xi_{1}(t), \xi_{2}(t)\right]^{T}$ satisfies $\sup _{t>0} E|\xi(t)|^{4}<K$, and one chooses $r_{1}=r_{2}=0.5$, $\mu_{1}=\mu_{2}=2, l_{10}=l_{20}=0.5, \lambda_{2}=v_{1}=v_{2}=0.5$. System (72) can be rewritten as

$$
\left\{\begin{array}{l}
\dot{x}_{1}=x_{2} d t+f_{1} d t+g_{1} \xi_{1} \\
\dot{x}_{2}=u d t+f_{2} d t+g_{2} \xi_{2} \\
y=x_{1}
\end{array}\right.
$$

where $f_{1}=-x_{1}, g_{1}=-\sqrt{\left|x_{1}\right|}, f_{2}=-x_{2}+x_{2}(t-\tau(t))+$ $x_{1}(t-\tau(t)), g_{2}=-\sqrt{\left|x_{2}\right|}$. One supposes that the state variables $x_{1}$ and $x_{2}$ are available. The random disturbance $\xi_{1}(t)$ and $\xi_{2}(t)$ are produced by

$$
\begin{aligned}
& \xi_{1}(t)=a_{1} \cos \left(\ell_{1} t+U_{1}\right) \sin \left(\bar{\ell}_{1} t\right), \\
& \xi_{2}(t)=a_{2} \cos \left(\ell_{2} t+U_{2}\right) \sin ^{2}\left(\bar{\ell}_{2} t\right)
\end{aligned}
$$

where $U_{1}$ and $U_{2}$ are random variables uniformly distributed on $[0,2 \pi]$ and $a_{1}, a_{2}, \ell_{1}, \ell_{2}, \bar{\ell}_{1}, \bar{\ell}_{2}$ are real constants, then $E \xi_{1}^{4}(t) \leq a_{1}^{4}$ and $E \xi_{2}^{4}(t) \leq a_{2}^{4}$. It is clear that (73) satisfies assumptions H0, $\mathbf{H 1}$ and $\mathbf{H} 2$.

In simulation, one chooses $a_{1}=1, a_{2}=1, \ell_{1}=1.2, \ell_{2}=$ $0.8, \bar{\ell}_{1}=1.5, \bar{\ell}_{2}=1, \pi_{1}=\pi_{2}=\pi_{21}=1$, and initial value $x_{1}(0)=0.5, x_{2}(0)=-0.5$. The responses of closedlooped system (73) and 40) are shown in Figure 1, which illustrates that the output regulation error of system (73) can be controlled in a small enough neighborhood of zero and the another state $x_{2}(t)$ is bounded in the mean-square sense.
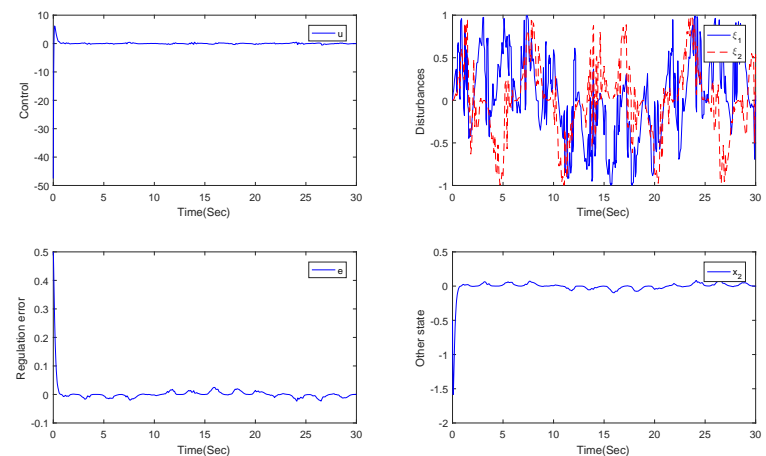

Figure 1. The responses of closed-loop system 73 and 401. 
Example 2: For the following random nonlinear feedback time-delay systems

$$
\left\{\begin{array}{l}
\dot{x}_{1}=x_{2}+\theta_{1} x_{1 t}^{2}+\theta_{1} x_{1} \xi_{1} \\
\dot{x}_{2}=u+\theta_{2} x_{1} \cos \left(x_{2 t}\right)+\theta_{2} x_{1 t} \sin \left(x_{2}\right) \xi_{2}, \\
y=x_{1}
\end{array}\right.
$$

where $x_{1 t}=x_{1}(t-\tau(t))$ and $x_{2 t}=x_{2}(t-\tau(t)), \tau(t)=$ $0.5(1+\cos t)$ that satisfies $0 \leq \tau(t) \leq 1$ and $\dot{\tau}(t) \leq 0.5$. Both $\theta_{1}$ and $\theta_{2}$ are unknown parameters. Only the state variable $x_{1}$ is measurable by output $y$, the state variable $x_{2}$ is unavailable. The random disturbance $\xi_{1}$ and $\xi_{2}$ are generated by 74 with $a_{1}=0.8, a_{2}=1.2, \ell_{1}=1, \ell_{2}=0.5, \bar{\ell}_{1}=0.5, \bar{\ell}_{2}=1$. So system (75) satisfies assumptions H0, H1' and H2'.

For simulation purpose, one selects $\theta_{1}=1.2$ and $\theta_{2}=$ 1.5 , and initial values $x_{1}(0)=0.01, x_{2}(0)=-0.3, \hat{x}_{1}(0)=$ $0.1, \hat{\theta}(0)=-0.5$, the design parameters $\mu=1, \kappa_{1}=1, b=2$, $d_{01}=1, d_{02}=0.1, d_{11}=d_{12}=1, d_{13}=0.1, d_{21}=d_{22}=1$, $d_{23}=0.1, c_{1}=1, c_{2}=1$. The responses of closed-loop system (75), 66) and 67) is given in Figure 2, which shows that the state $x_{2}$ is bounded in the mean-square sense and the output regulation error $e$ can be made small enough in the mean-square sense.
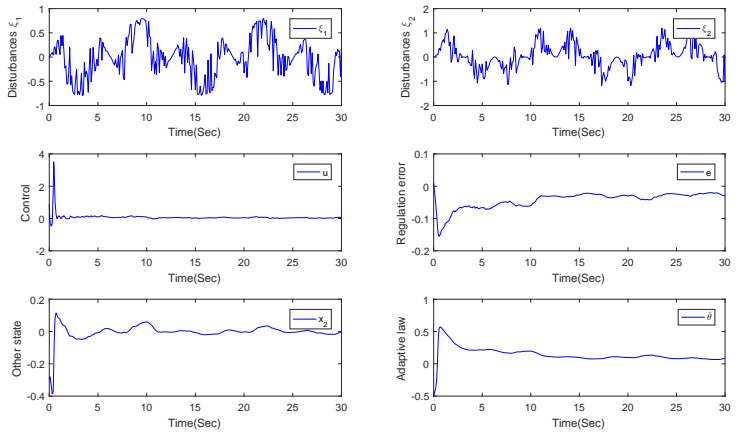

Figure 2. The responses of closed-loop system 75 , 66) and 677.

\section{CONCLUSION}

This paper investigates the stability of random nonlinear systems with time-delay and its application in regulation control. The existence and uniqueness of solution to random nonlinear time-delay systems is guaranteed by proposed conditions. Next, some criteria on noise-to-state stability of random nonlinear systems with time-delay are established. As applications, two kinds of regulation control problems are solved by state feedback method and adaptive output feedback method.

Many related problems deserves further investigation. For instance, how to apply the obtained results in stability analysis and synthesis of practical systems with time-delay, such as Hamiltonian mechanical systems and Lagrangian mechanical system; how to generalize this result to random switched systems with time-delay.

\section{APPENDIX A}

The Proof of 37]

Proof: From $\mathbf{H 2}$ and (28), one can obtain the following inequality

$$
\begin{aligned}
& z_{i} f_{i}-z_{i} \sum_{j=1}^{i-1} \frac{\partial \alpha_{i-1}}{\partial x_{j}}\left(x_{j+1}+f_{j}\right) \\
& \leq\left|z_{i}\right|\left(\bar{\theta} \sum_{l=1}^{i}\left(\left|x_{l}\right|+\left|x_{l}(t-\tau(t))\right|\right)\right. \\
& \quad+\sum_{j=1}^{i-1} \Xi_{j(i-1)}\left(\left|x_{j+1}\right|+\bar{\theta} \sum_{l=1}^{j}\left(\left|x_{l}\right|+\left|x_{l}(t-\tau(t))\right|\right)\right) \\
& \leq\left|z_{i}\right| \eta_{i}\left(\sum_{l=1}^{i}\left(\left|x_{l}\right|+\left|x_{l}(t-\tau(t))\right|\right)+\sum_{j=1}^{i-1}\left|x_{j+1}\right|\right. \\
& \left.\quad+\sum_{j=1}^{i-1} \sum_{l=1}^{j}\left(\left|x_{l}\right|+\left|x_{l}(t-\tau(t))\right|\right)\right) \\
& =\left|z_{i}\right| \eta_{i}\left(\sum_{l=2}^{i}(i+2-l)\left|x_{l}\right|\right. \\
& \left.\quad+\sum_{l=1}^{i}(i+1-l)\left|x_{l}(t-\tau(t))\right|+i\left|x_{1}\right|\right) \\
& \leq\left|z_{i}\right| \bar{\eta}_{i}\left(\left|z_{1}\right|+\sum_{l=2}^{i}\left(\left|z_{l}\right|+\beta_{l-1}\left|z_{l-1}\right|\right)+\left|z_{1}(t-\tau(t))\right|\right. \\
& \left.\quad+\sum_{l=2}^{i}\left(\left|z_{l}(t-\tau(t))\right|+\beta_{l-1}\left|z_{l-1}(t-\tau(t))\right|\right)\right) \\
& \leq\left|z_{i}\right| \tilde{\eta}_{i}\left(\sum_{l=1}^{i-1}\left(\left|z_{l}\right|+\left|z_{l}(t-\tau(t))\right|\right)\right. \\
& +\left|z_{i}\right| \bar{\eta}_{i}\left(\left|z_{i}\right|+\left|z_{i}(t-\tau(t))\right|\right),
\end{aligned}
$$

where $\eta_{i}=\max _{1 \leq j \leq i-1}\left\{\bar{\theta}, \Xi_{j(i-1)}, \Xi_{j(i-1)} \bar{\theta}\right\}, \bar{\eta}_{i}=\eta_{i} i, \tilde{\eta}_{i}=$ $\bar{\eta}_{i} \max _{1 \leq l \leq i-1}\left\{1+\beta_{l}\right\}, i=2,3, \cdots, n$.

By Young's inequality, one gets

$$
\begin{aligned}
\left|z_{i}\right| \tilde{\eta}_{i} \sum_{l=1}^{i-1}\left|z_{l}\right| \leq & \frac{(i-1)}{2} \tilde{\eta}_{i}^{2} z_{i}^{2}+\sum_{l=1}^{i-1} \frac{1}{2} z_{l}^{2} \\
\left|z_{i}\right| \bar{\eta}_{i}\left|z_{i}(t-\tau(t))\right| \leq & \frac{e^{-\tau}\left(1-\tau^{*}\right)}{2} z_{i}^{2}(t-\tau(t)) \\
& +\frac{e^{\tau}}{2\left(1-\tau^{*}\right)} \bar{\eta}_{i}^{2} z_{i}^{2} \\
\left|z_{i}\right| \tilde{\eta}_{i} \sum_{l=1}^{i-1}\left|z_{l}(t-\tau(t))\right| \leq & \frac{e^{-\tau}\left(1-\tau^{*}\right)}{2} \sum_{l=1}^{i-1} z_{l}^{2}(t-\tau(t)) \\
& +\frac{(i-1) e^{\tau}}{2\left(1-\tau^{*}\right)} \tilde{\eta}_{i}^{2} z_{i}^{2}
\end{aligned}
$$

Substituting A.2 -A.4 to A.1 leads

$$
\begin{aligned}
& z_{i}\left(f_{i}-\sum_{j=1}^{i-1} \frac{\partial \alpha_{i-1}}{\partial x_{j}}\left(x_{j+1}+f_{j}\right)\right) \\
\leq & \sum_{l=1}^{i-1} z_{l}^{2}+\frac{e^{-\tau}\left(1-\tau^{*}\right)}{2} \sum_{l=1}^{i} z_{l}^{2}(t-\tau(t))+\Omega_{i} z_{i}^{2},
\end{aligned}
$$


where $\Omega_{i}=\frac{(i-1)}{2} \tilde{\eta}_{i}^{2}+\frac{(i-1)}{2\left(1-\tau^{*}\right)} e^{\tau} \tilde{\eta}_{i}^{2}+\bar{\eta}_{i}+\frac{1}{2\left(1-\tau^{*}\right)} e^{\tau} \bar{\eta}_{i}^{2}$ is a positive constant.

\section{APPENDIX B}

The Proof of (38)

Proof: It follows from $\mathbf{H} 2$ and (28) that

$$
\begin{aligned}
& z_{i} g_{i} \xi_{i}-z_{i} \sum_{j=1}^{i-1} \frac{\partial \alpha_{i-1}}{\partial x_{j}} g_{j} \xi_{j} \\
& \leq \frac{1}{2}\left|z_{i}\right|\left(g_{i}^{2}+\left|\xi_{i}\right|^{2}\right)+\frac{1}{2}\left|z_{i}\right| \sum_{j=1}^{i-1} \Xi_{j(i-1)}\left(g_{j}^{2}+\left|\xi_{j}\right|^{2}\right) \\
& \leq\left|z_{i}\right| \zeta_{i}\left(\sum_{l=1}^{i}\left(\left|x_{l}\right|+\left|x_{l}(t-\tau(t))\right|\right)\right. \\
& \left.+\sum_{j=1}^{i-1} \sum_{l=1}^{j}\left(\left|x_{l}\right|+\left|x_{l}(t-\tau(t))\right|\right)\right) \\
& +\frac{1}{2}\left|z_{i}\right|\left(\left|\xi_{i}\right|^{2}+\sum_{j=1}^{i-1} \Xi_{j(i-1)}\left|\xi_{j}\right|^{2}\right) \\
& =\left|z_{i}\right| \zeta_{i} \sum_{l=1}^{i}(i-l+1)\left(\left|x_{l}\right|+\left|x_{l}(t-\tau(t))\right|\right) \\
& +\frac{1}{2}\left|z_{i}\right|\left(\left|\xi_{i}\right|^{2}+\sum_{j=1}^{i-1} \Xi_{j(i-1)}\left|\xi_{j}\right|^{2}\right) \\
& \leq\left|z_{i}\right| \bar{\zeta}_{i} \sum_{l=1}^{i}\left(\left|x_{l}\right|+\left|x_{l}(t-\tau(t))\right|\right) \\
& +\frac{1}{2}\left|z_{i}\right|\left(\left|\xi_{i}\right|^{2}+\sum_{j=1}^{i-1} \Xi_{j(i-1)}\left|\xi_{j}\right|^{2}\right) \\
& \leq\left|z_{i}\right| \bar{\zeta}_{i}\left(\left|z_{1}\right|+\left|z_{1}(t-\tau(t))\right|+\sum_{l=2}^{i}\left(\left|z_{l}\right|+\beta_{l-1}\left|z_{l-1}\right|\right)\right. \\
& \left.+\sum_{l=2}^{i}\left(\left|z_{l}(t-\tau(t))\right|+\beta_{l-1}\left|z_{l-1}(t-\tau(t))\right|\right)\right) \\
& +\frac{1}{2}\left|z_{i}\right|\left(\left|\xi_{i}\right|^{2}+\sum_{j=1}^{i-1} \Xi_{j(i-1)}\left|\xi_{j}\right|^{2}\right) \\
& \leq\left|z_{i}\right| \tilde{\zeta}_{i}\left(\sum_{l=1}^{i-1}\left(\left|z_{l}\right|+\left|z_{l}(t-\tau(t))\right|\right)\right. \\
& +\left|z_{i}\right| \bar{\zeta}_{i}\left(\left|z_{i}\right|+\left|z_{i}(t-\tau(t))\right|\right) \\
& +\frac{1}{2}\left|z_{i}\right|\left(\left|\xi_{i}\right|^{2}+\sum_{j=1}^{i-1} \Xi_{j(i-1)}\left|\xi_{j}\right|^{2}\right),
\end{aligned}
$$

where $\zeta_{i}=\max _{1 \leq j \leq i-1}\left\{\frac{1}{2} \bar{\theta}, \frac{1}{2} \bar{\theta} \Xi_{j(i-1)}\right\}, \bar{\zeta}_{i}=\zeta_{i} i, \tilde{\zeta}_{i}=$ $\bar{\zeta}_{i} \max _{1 \leq l \leq i-1}\left\{1+\beta_{l}\right\}, i=2,3, \cdots, n$.

By Young's inequality, one obtains

$$
\begin{aligned}
\frac{1}{2}\left|z_{i}\right|\left|\xi_{i}\right|^{2} & \leq \frac{1}{16 \pi_{i}} z_{i}^{2}+\pi_{i}\left|\xi_{i}\right|^{4}, \\
\left|z_{i}\right| \tilde{\zeta}_{i} \sum_{l=1}^{i-1}\left|z_{l}\right| & \leq \frac{(i-1)}{2} \tilde{\zeta}_{i}^{2} z_{i}^{2}+\sum_{l=1}^{i-1} \frac{1}{2} z_{l}^{2},
\end{aligned}
$$

$$
\begin{aligned}
\frac{1}{2}\left|z_{i}\right| \sum_{j=1}^{i-1} \Xi_{j(i-1)}\left|\xi_{j}\right|^{2} \leq & \sum_{j=1}^{i-1} \frac{1}{16 \pi_{i j}} \Xi_{j(i-1)}^{2} z_{i}^{2} \\
& +\sum_{j=1}^{i-1} \pi_{i j}\left|\xi_{j}\right|^{4} \\
\left|z_{i}\right| \bar{\zeta}_{i}\left|z_{i}(t-\tau(t))\right| \leq & \frac{e^{-\tau}\left(1-\tau^{*}\right)}{2} z_{i}^{2}(t-\tau(t)) \\
& +\frac{e^{\tau}}{2\left(1-\tau^{*}\right)} \bar{\zeta}_{i}^{2} z_{i}^{2} \\
\left|z_{i}\right| \tilde{\zeta}_{i} \sum_{l=1}^{i-1}\left|z_{l}(t-\tau(t))\right| \leq & \frac{e^{-\tau}\left(1-\tau^{*}\right)}{2} \sum_{l=1}^{i-1} z_{l}^{2}(t-\tau(t)) \\
& +\frac{(i-1) e^{\tau}}{2\left(1-\tau^{*}\right)} \tilde{\zeta}_{i}^{2} z_{i}^{2} .
\end{aligned}
$$

Substituting (B.2)-B.6 to (B.1) yields

$$
\begin{aligned}
& z_{i} g_{i} \xi_{i}-z_{i} \sum_{j=1}^{i-1} \frac{\partial \alpha_{i-1}}{\partial x_{j}} g_{j} \xi_{j} \\
\leq & \sum_{l=1}^{i-1} z_{l}^{2}+\Lambda_{i} z_{i}^{2}+\pi_{i}\left|\xi_{i}\right|^{4}+\sum_{j=1}^{i-1} \pi_{i j}\left|\xi_{j}\right|^{4} \\
& +\frac{e^{-\tau}\left(1-\tau^{*}\right)}{2} \sum_{l=1}^{i} z_{l}^{2}(t-\tau(t)),
\end{aligned}
$$

where $\Lambda_{i}=\frac{(i-1)}{2} \tilde{\zeta}_{i}^{2}+\frac{(i-1)}{2\left(1-\tau^{*}\right)} e^{\tau} \tilde{\zeta}_{i}^{2}+\bar{\zeta}_{i}+\frac{1}{2\left(1-\tau^{*}\right)} e^{\tau} \bar{\zeta}_{i}^{2}+\frac{1}{16 \pi_{i}}+$ $\sum_{j=1}^{i-1} \frac{1}{16 \pi_{i j}} \Xi_{j(i-1)}^{2}$ is a positive constant.

\section{REFERENCES}

[1] M. Malek-Zavarei and M. Jamshidi, Time-Delay Systems: Analysis, Optimization and Applications, Amsterdam, Holland: Elsevier Science Publishers, 1987.

[2] K. Gu, V. L. Kharitonov, and J. Chen, Stability of Time-Delay Systems, Boston, USA: Birkhäuser, 2003.

[3] M. Lakshmanan and D.V. Senthilkumar, Dynamics of Nonlinear TimeDelay Systems, Berlin, Germany: Springer-Verlag, 2010.

[4] T. T. Soong, Random Differential Equations in Science and Engineering, New York, USA: Academic Press, 1973.

[5] R. Z. Khas'minskii, Stochastic Stability of Differential Equations, Rockville, MD, USA: Sijthoff \& Noordhoff International Publishers, 1980.

[6] A. M. Samojlenko and O. Stanzhytskyi, Qualitative and Asymptotic Analysis of Differential Equations with Random Perturbations, Singapore: World Scientific Publishing, 2011.

[7] Z. J. Wu, "Stability criteria of random nonlinear systems and their applications," IEEE Transactions on Automatic Control, vol. 60, no. 4, pp. 1038-1049, 2015.

[8] M. Krstić and H. Deng, Stabilization of Nonlinear Uncertain Systems, New York, USA: Springer, 1998.

[9] H. Deng, M. Krstić, and R. J. Williams, "Stabilization of stochastic nonlinear systems driven by noise of unknown covariance," IEEE Transactions on Automatic Control, vol. 46, no. 8, pp. 1237-1253, 2001.

[10] X. R. Mao, Stochastic Differential Equations and Applications(2nd ed.), Chichester, UK: Horwood Publishing Limited, 2007.

[11] Y. S. Fu, Z. H. Tian, and S. J. Shi, "Output-feedback stabilization for a class of stochastic time-delay nonlinear systems," IEEE Transactions on Automatic Control, vol. 50, no. 6, pp. 847-850, 2005.

[12] S. J. Liu, S. Z. Ge, and J. F. Zhang, "Adaptive output-feedback control for a class of uncertain stochastic nonlinear systems with time delays," International Journal of Control, vol. 81, no. 8, pp. 1210-1220, 2008.

[13] L. Liu and X. J. Xie, "Output-feedback stabilization for stochastic high-order nonlinear systems with time-varying delay," Automatica, vol. 47, no. 12, pp. 2772-2779, 2011. 
[14] L. Liu and X. J. Xie, "State feedback stabilization for stochastic feedforward nonlinear systems with time-varying delay," Automatica, vol. 49, no. 4, pp. 936-942, 2013.

[15] D. F. Zhang, Z. J. Wu, X. M. Sun, and W. Wang, "Noise-tostate stability for a class of random systems with state-dependent switching," IEEE Transactions on Automatic Control, vol. 61, no. 10, pp. 3164-3170, 2016.

[16] H. Zhang, Y. Q. Xia, and Z. J. Wu, "Noise-to-state stability of random switched systems and its applications," IEEE Transactions on Automatic Control, vol. 61, no. 6, pp. 1607-1612, 2016.

[17] T. C. Jiao, W. X. Zheng, and S. Y. Xu, "On stability of a class of switched nonlinear systems subject to random disturbances," IEEE Transactions on Circuits and Systems I: Regular Papers, vol. 63, no. 12, pp. 2278-2289, 2016.

[18] Z. J. Wu, S. T. Wang, and M. Y. Cui, "Tracking controller design for random nonlinear benchmark system," Journal the Franklin Institute, vol. 354, no. 1, pp. 360-371, 2017.

[19] L. Q. Yao and W. H. Zhang, "Adaptive tracking control for a class of random pure-feedback nonlinear systems with Markovian switching," International Journal of Robust and Nonlinear Control, vol. 28, no. 8, pp. 3112-3126, 2018.

[20] F. C. Klebaner, Introduction to Stochastic Calculus with Applications(2nd ed.), London, UK: Imperial College Press, 2005.

[21] T. M. Flett, Differential analysis, Cambridge, UK: Cambridge University Press, 1980.

[22] G. H. Hardy, J. E. Littlewood, and G. Polya, Ineqaulities(2nd ed.), Cambridge, UK: Cambridge University Press, 1989.

[23] C. C. Hua, P. X. Liu, and X. P. Guan, "Backstepping control for nonlinear systems with time delays and applications to chemical reactor systems," IEEE Transactions on Industrial Electronics, vol. 56, no. 9, pp. 3723-3732, 2009.

[24] W. S. Chen, J. Wu, and L. C. Jiao, "State-feedback stabilization for a class of stochastic time-delay nonlinear systems," International Journal of Robust and Nonlinear Control, vol. 22, no. 17, pp. 19211937, 2012. 